\title{
Independent and Interacting Value Systems for Reward and Information in the Human Brain
}

\author{
Authors: I. Cogliati Dezza ${ }^{1,2,3 *}$, A. Cleeremens ${ }^{1}$, W. Alexander ${ }^{3,4}$
}

\section{Affiliations:}

$7 \quad{ }^{1}$ Center for Research in Cognition \& Neurosciences, ULB Neuroscience Institute, Université Libre de

8 Bruxelles, Brussels, Belgium

$9{ }^{2}$ Department of Experimental Psychology, Faculty of Brain Sciences, University College London,

10 London, UK

$11{ }^{3}$ Department of Experimental Psychology, Ghent University, Ghent, Belgium

$12{ }^{4}$ Center for Complex Systems and Brain Sciences, Florida Atlantic University, USA

13

$14 *$ Correspondence to: $\underline{\text { irene.cogliatidezza@gmail.com. }}$

15

One Sentence Summary: Reward and information value are independently optimized in the Human PFC and are then combined in subcortical regions in order to implement choices. 


\section{ABSTRACT}

21 Theories of Prefrontal Cortex (PFC) as optimizing reward value have been widely deployed to explain its

22 activity in a diverse range of contexts, with substantial empirical support in neuroeconomics and decision

23 neuroscience. Theoretical frameworks of brain function, however, suggest the existence of a second,

24 independent value system for optimizing information during decision-making. To date, however, there has

25 been little direct empirical evidence in favor of such frameworks. Here, by using computational modeling,

26 model-based fMRI analysis, and a novel experimental paradigm, we aim at establishing whether

27 independent value systems exist in human PFC. We identify two regions in the human PFC which

28 independently encode distinct value signals. These value signals are then combined in subcortical regions

29 in order to implement choices. Our results provide empirical evidence for PFC as an optimizer of

30 independent value signals during decision-making under realistic scenarios, with potential implications for

31 the interpretation of PFC activity in both healthy and clinical population. 


\section{INTRODUCTION}

34 A general organizational principle of reward value computation and comparison in PFC has accrued 35 widespread empirical support in neuroeconomics and decision neuroscience ${ }^{1-3}$. According to this account, 36 the relative reward value of immediate, easily-obtained, or certain outcomes positively contributes to the 37 net-value of a choice ${ }^{4} 5$, while delay, difficulty, cost or uncertainty in realizing prospective outcomes 38 negatively contribute to it ${ }^{6-8}$ (Figure 1A). Although substantial empirical evidence supports the interpretation of PFC function as a single distributed system that performs a cost-benefit analysis in order to optimize the net value of rewards ${ }^{1-3}{ }^{9}$, other perspectives have suggested the existence of a second, independent value system for optimizing information within PFC.

Unifying theories of brain organization and function propose that information gain plays a similar role as does reward in jointly minimizing surprise ${ }^{10-12}$, allowing a behaving agent to better anticipate environmental contingencies. Some reinforcement learning (RL) frameworks distinguish extrinsicallymotivated (reward-based) behavior from intrinsically-motivated behavior to explain phenomena such as curiosity ${ }^{13}$, directed exploration ${ }^{14}$, and play ${ }^{15}$ in the absence of explicit reward. More broadly, results from machine learning demonstrate that explicitly incorporating information optimization in choice behavior can dramatically improve performance on complex tasks ${ }^{16,17}$. Altogether these perspectives suggest that information is intrinsically valuable ${ }^{18} 19$ and positively contributes to the net-value computation ${ }^{20}{ }^{21}$ during decision-making (Figure 1B). To date, however, direct neural evidence for such a system is lacking. Using computational modeling, model-based fMRI analyses, and a novel experimental paradigm, we aim at establishing whether a dedicated and independent value system for information exists in human PFC. Individuating independent value signals optimized by PFC can offer insight into many disorders characterized by both reward and information-seeking abnormalities such as schizophrenia ${ }^{22}$, depression ${ }^{23}$ and addiction ${ }^{24}$.

Simulations of an RL model which consists of independent value systems ${ }^{25}$ independently optimizing 57 information and reward demonstrate how reward-focused fMRI analyses ${ }^{26-30}$ may be unsuccessful at 58 identifying an independent information value system as a consequence of correlated activity (Figure 1). In 59 such systems, independently optimizing reward and information entails a tradeoff: optimizing reward 60 means not optimizing information, and vice-versa. In other words, even if reward and information systems 61 are independent, they are nonetheless (negatively) correlated through behavior ${ }^{20}$. This correlation is 62 consistent across different decision-making tasks ${ }^{20,27,30}$ (Figure 1; Supplement; Figure S1), and is also 63 observed in single-value system models (Figure 1 C,D). Crucially, the results of our simulations imply that 
reward-focused univariate fMRI analyses ${ }^{26-30}$ (which uniquely focus on the reward dimension) may misattribute information value to a system computing costs (diminishing reward value), rather than to an independent information value system.

To test whether a dedicated and independent value system for information exists in human PFC, we focus on two regions, ventromedial PFC (vmPFC) and dorsal Anterior Cingulate Cortex (dACC), which are frequently identified as calculating the positive (vmPFC) and negative (dACC) components of a costbenefit analysis. Activity observed in vmPFC and dACC frequently exhibits a pattern of symmetric opposition: as dACC activity increases, vmPFC activity decreases- a pattern that holds across a wide range of value-based decision-making contexts, including foraging ${ }^{27,30}$, risk $^{31}$, intertemporal ${ }^{32,33}$ and effort-based choice $^{34,35}$ (Table S1). The variety of contexts in which this pattern is observed suggests a general role for these regions in contributing to the net-value associated with a choice ${ }^{1-3}$, with vmPFC positively and dACC negatively contributing to the net-value computation. Even for studies in which dACC and vmPFC activity is dissociated 3637389394028 activity in vmPFC is generally linked to reward value, while activity in dACC is often interpreted as indexing negative or non-rewarding attributes of a choice (including ambiguity ${ }^{41}$ difficulty ${ }^{28}$, negative reward value ${ }^{35}$, cost and effort ${ }^{42}$ ). Model simulations further demonstrate that functional opposition between reward and information system in value-based choices may be observed even in absence of a clear symmetric opposition of activity (Supplement; Figure S2). The interpretation of $\mathrm{dACC}$ and vmPFC as opposing one another therefore includes both symmetrically-opposed activity, as well as a more general functional opposition in value-based decision-making.

83 Here, we used the apparent symmetrical opposition between $\mathrm{AACC}$ and vmPFC as a tool to investigate the 84 existence of a dedicated and independent value system for information in human PFC. Simulations of two 85 computational models (methods), one with a single value system (Figure $1 \mathbf{C , D}$ ) and one with independent 86 value systems (Figure $1 \mathbf{E}$ ), demonstrate opposed activity for reward value and information gain when 87 these two factors are confounded. Under the single value system model, symmetrically-opposed activity should still be observed after controlling for the confound (Figure 1 F,G). In contrast, under the 
A Single Value System

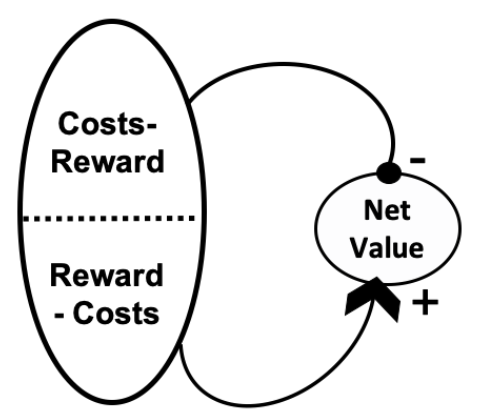

B Dual Value System

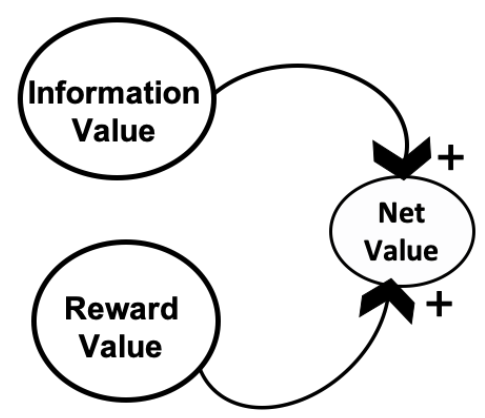

Symmetrical Activity

C D Cost-Reward

E

Dual Value System

Reward System

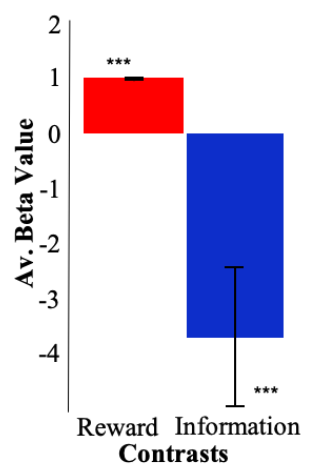

F

\section{Reward-Cost}

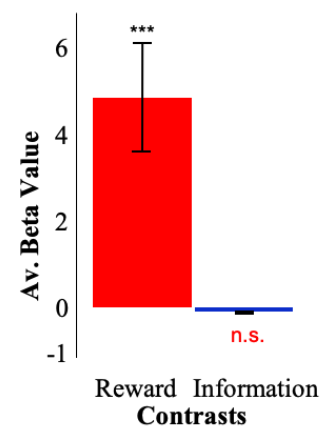

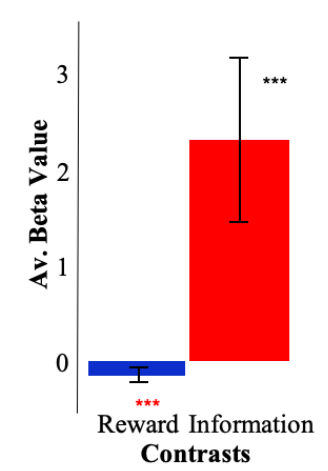

Controlling for correlated variables

G

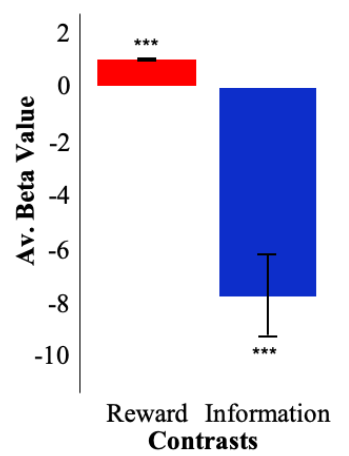

Contrasts
Cost-Reward

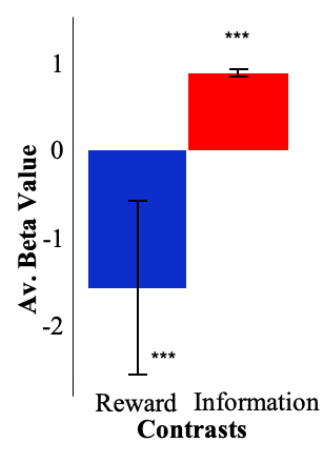

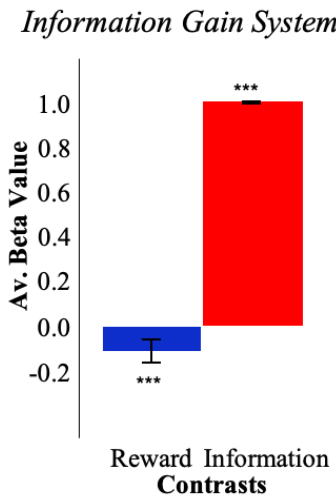

\section{H Dual Value System}

Reward System

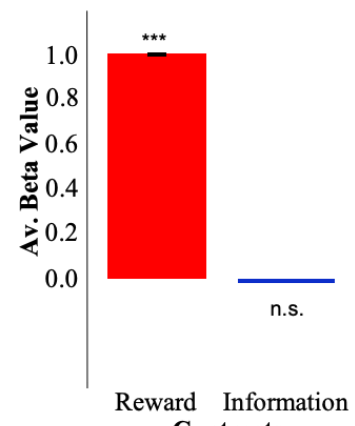

Contrasts
Information Gain System

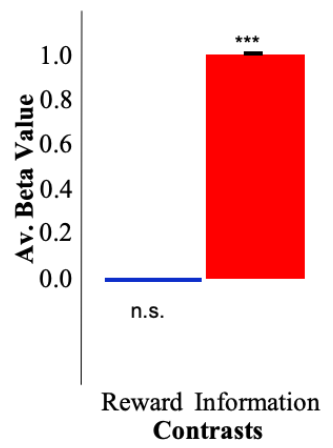

96 (A) In the single value system framework costs (negative information value) and rewards interact to produce a net-value estimate,

97 (B) while in the dual value system framework information value and reward value are estimated independently. When no controlling

98 for correlations, $(\mathbf{C})$ in the single reward value system (Reward-Cost) and (D) in the single information value system (Cost-Reward)

99 components representing costs and rewards are represented following an opposing symmetrical pattern of activity. This pattern is 
100 also observed in the dual value system, despite the independence of information and reward systems in this framework: optimizing

101 either reward or information gain is associated with decreased activity in the alternate value system, leading to symmetrically

102 opposed activity between the systems (E). When controlling for correlations, symmetrical activity is still observed in the single

103 information value system $(\mathbf{G})$, while it disappears in both single reward value system $(\mathbf{F})$ and dual value system $(\mathbf{H})$.

104 


\section{RESULTS}

\section{Reward and information jointly influence choices}

107 Human participants made sequential choices among 3 decks of cards over 128 games, receiving between 1 108 and 100 points after each choice (Figure 2; Methods). The task consisted of two phases (Figure 2A): a 109 learning phase (i.e., forced-choice task) in which participants were instructed which deck to select on each 110 trial (Figure 2B), and a decision phase (i.e., free-choice task) in which participants made their own choices 111 with the goal of maximizing the total number of points obtained at the end of the experiment (Figure 2C). 112 By carefully controlling for reward and information delivered to participants in the learning phase, in this 113 gambling task it is possible to orthogonalize available information and reward delivered to participants in 114 the first free choice trials by clustering choices in specific task conditions ${ }^{20}$ (Methods). To have a better 115 estimate of the neural activity over the overall performance, however, we adopted trial-by-trial fMRI 116 analyses. This introduces information-reward confound in our analysis. Logistic regression of subjects' 117 behavior on the first trial shows that indeed choices were driven both by the reward $(3.22, \mathrm{t}(1,19)=12.4, p$ $\left.118<10^{-9}\right)$ and information $\left(-3.68, \mathrm{t}(1,19)=-7.84, p<10^{-6}\right)$ experienced during the learning phase (Figure 119 2D). In all our analyses, we took care of this confound by using dedicated model-based approaches 120 (Methods).

\section{The gambling task elicits activity in dACC and vmPFC}

122 We first investigated whether our gambling task elicits dACC and vmPFC activity. We conducted a one 123 sample t-test on the beta weights estimated for GLMO which consists of two regressors, one modelling 124 choice onset associated with selection of the highest rewarded options (Highest Reward), and another 125 regressor modelling choice onset associated with lower rewarded options (Lower Reward). This and all 126 subsequent fMRI analyses focus on the time window preceding the first free choices (Methods). Results 127 showed that vmPFC activity is positively correlated with the reward associated with the chosen option 128 (Highest reward - Lower Reward; FEW p $=0.009$, uncorr $\mathrm{p}=0.000$, voxel extent $=203$, peak voxel 129 coordinates $(-6,30,-14), \mathrm{t}(19)=5.48$; Figure $2 \mathbf{H})$, while dACC/preSMA activity was negatively correlated 130 with the reward of the chosen option (Lower Reward-Highest Reward; FEW p $=0.158$, uncorr $\mathrm{p}=0.014$, 131 voxel extent $=87$, peak voxel coordinates $(-2,12,58), \mathrm{t}(19)=4.66$; Figure 2G). We note that the cluster 132 of activity we identify as "dACC" spans into supplementary motor areas. Many fMRI studies on value133 based decision-making reporting similar activity patterns, however, commonly refer to activity around this 134 area as dACC. Additionally, in the Lower Reward - Highest Reward contrast activity did not survive 135 correction for multiple comparisons. This might be due to individual differences in subjective reward value. 
136 We address this issue in the next section by adopting model-based approaches. Here, we repeated the same 137 analysis by selecting a small volume image including the regions that were surviving at uncorr $\mathrm{p} 0.001$. 138 Results show a significant cluster at voxel coordinates $(-2,12,58)$ after correcting for multiple comparisons 139 (FEW $\mathrm{p}=0.011)$. Overall, these results indicate that our gambling task elicits activity in dACC and vmPFC 140 and this activity follows a symmetrically opposite pattern (Figure 2E).

A $\mathrm{N}^{\circ}$ Game: 1

Forced Choice Task
\begin{tabular}{|l|l|l|l|l|l|l|l|l|l|l|l|} 
Free Choice Task \\
\hline T1 & T2 & T3 & T4 & T5 & T6 \\
\hline
\end{tabular}

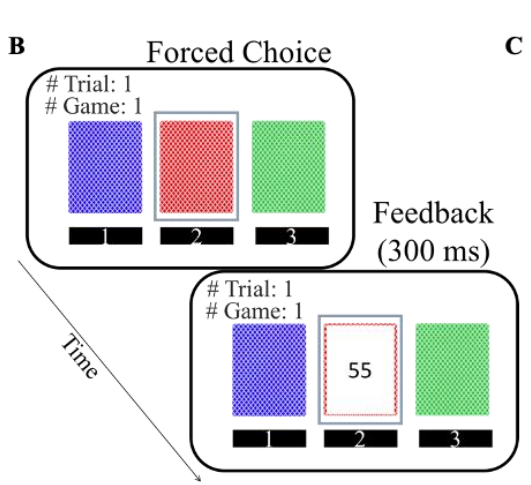

C

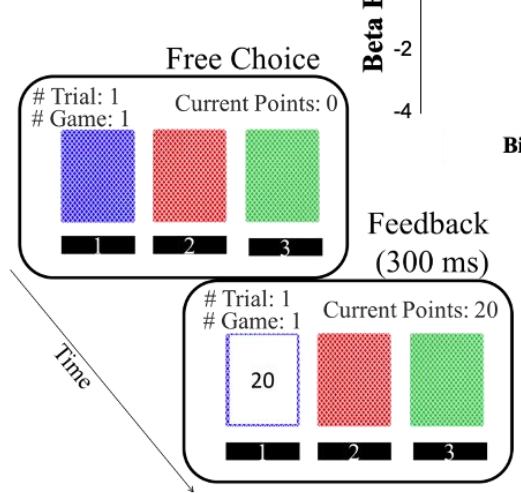

F

\begin{tabular}{ll}
\hline \multicolumn{1}{c}{ Main GLMs } \\
\hline GLM0 & Classical Univariate Analysis \\
\hline GLM1 & Model-Based Reward \\
\hline GLM2 & Model-Based Information \\
\hline GLM3 & Model-Based Reward (Controlling for Information) \\
\hline GLM4 & Model-Based Information (Controlling for Reward) \\
\hline GLM5 & $\begin{array}{l}\text { Model-Based Information (Controlling for Instrumental } \\
\text { Information) }\end{array}$ \\
\hline GLM8 & Model-Based Response Probability \\
\hline
\end{tabular}

G

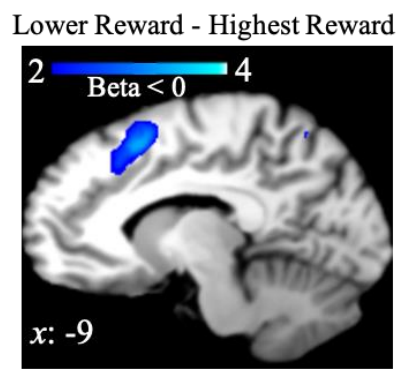

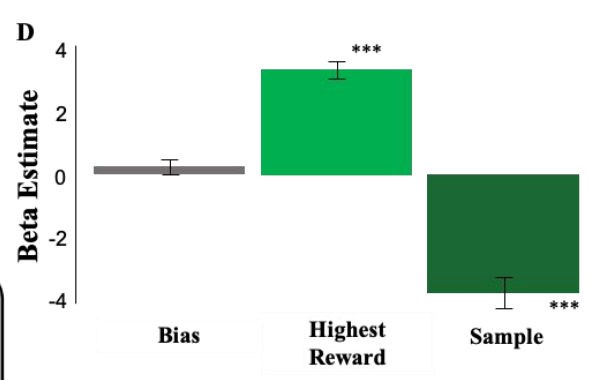

$\mathbf{E}$

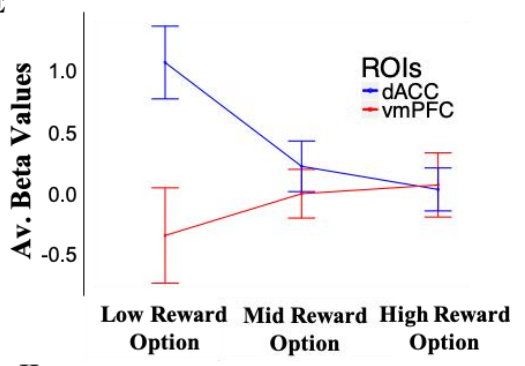

H

Highest Reward - Lower Reward

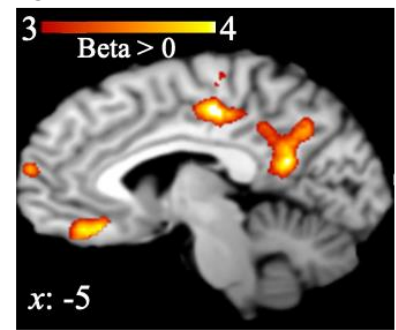

(A) One game of the behavioral task consisted of 6 consecutive forced choice trials and from 1 to 6 free-choice trials. FMRI analyses focused on the first free-choice trial (shown in yellow) in which reward and information were decorrelated. (B) In the forced-choice task participants chose a pre-selected deck of cards (outlined in blue). (C) In the free-choice task they were instead free to choose a deck of cards in order to maximize the total number of points. (D) Participants' behavior was driven by both experienced reward and the number of times the options were chosen in previous trials (beta weights from a logistic regression; dependent variable is participants' exploitative choices). (E) DACC and vmPFC activities follow a symmetrical opposite pattern. Activity is split as a function of reward levels (low, mid and high). (F) Types of GLMs adopted in the fMRI analysis. (G) DACC activity related to selecting the lower reward option. (H) VmPFC related to selecting the highest reward option. Activity scale represents z-score. 
154 In the previous section, we showed that our gambling task elicits both dACC and vmPFC activity in a

155 symmetrically opposed pattern. Here, we tested whether 1) this symmetrical activity relates to both reward

156 and information signals and, 2) whether the symmetrically opposite pattern is the product of correlated

157 variables.

158 We fitted a reinforcement learning (RL) model with information integration ${ }^{25}$ to participants' behavior to 159 obtain subjective evaluations of reward and information (Methods). Subjective evaluations of reward were 160 computed as relative reward values (RelReward; Methods) as it has been shown that vmPFC represents the 161 relative expected reward of the chosen option ${ }^{28}$ (Supplement). We additionally considered alternative 162 ways in which vmPFC might compute reward signals. This includes expected reward values, the maximum 163 value of 3 decks, the minimum value of the 3 decks, the reward value variation for the chosen option, the 164 averaged value of the 3 decks, and the value of the chosen option minus the value of the best second option 165 (Supplement). These analyses suggested that RelReward best describes vmPFC activity. We therefore 166 adopted this computation in the following analyses. Subjective evaluations of information were computed 167 as the prospective information gain for selecting a deck, derived from behavioral fits of our RL model to 168 subject data (Information Gain; Methods). Alternative computations of information (e.g., the standard 169 deviation of the reward distribution of the chosen option) were highly correlated with this regressor (for 170 most of the subjects $r>0.6$ ) and were therefore not considered any further. RelReward and Information 171 Gain refer to the value associated with the chosen option in the first free-choice trial before the feedback 172 was delivered (Methods).

173 We then regressed RelReward and Information Gain on the BOLD signal recorded on the first free-choice 174 trial of each game. RelReward and Information Gain were used as the only parametric modulators in 175 separate GLMs to identify BOLD activity related to reward (GLMI) and to information (GLM2) 176 respectively, on the first free-choice trial (Figure 2F). Unless otherwise specified, all results for these and 177 subsequent analyses are cluster-corrected with a voxel-wise threshold of 0.001 . Activity in vmPFC on the 178 first free-choice trial correlated positively with RelReward (FWE $p<0.001$, voxel extent $=1698$, peak 179 voxel coordinates $(8,28,-6), \mathrm{t}(19)=6.62)($ Figure 3A) and negatively with Information Gain $($ FWE $p<$ 180 0.001, voxel extent $=720$, peak voxel coordinates $(-10,28,-2), \mathrm{t}(19)=5.36)$ (Figure 3B), while activity 181 in dACC was negatively correlated with RelReward (FWE $p=0.001$, voxel extent $=321$, peak voxel 182 coordinates $(6,24,40), \mathrm{t}(19)=4.59$; Figure 3A) and positively with Information Gain (FWE $p<0.001$, 183 voxel extent $=1441$, peak voxel coordinates $(8,30,50), \mathrm{t}(19)=7.13$; Figure 3B). A similar symmetrical 184 opposition was observed when we considered expected reward values, instead of relative reward values, 185 for the reward dimension (Supplement). Directly contrasting the beta estimates for Information Gain and 
RelReward in both clusters revealed a symmetrically-opposed pattern of activity in both dimensions (Figure 3C). Additionally, we observed that the beta values for GLM1 and GLM2 were negatively correlated across subjects for both the vmPFC cluster (Figure 3D) and the dACC cluster (Figure 3E). along both the information and reward dimension. variables
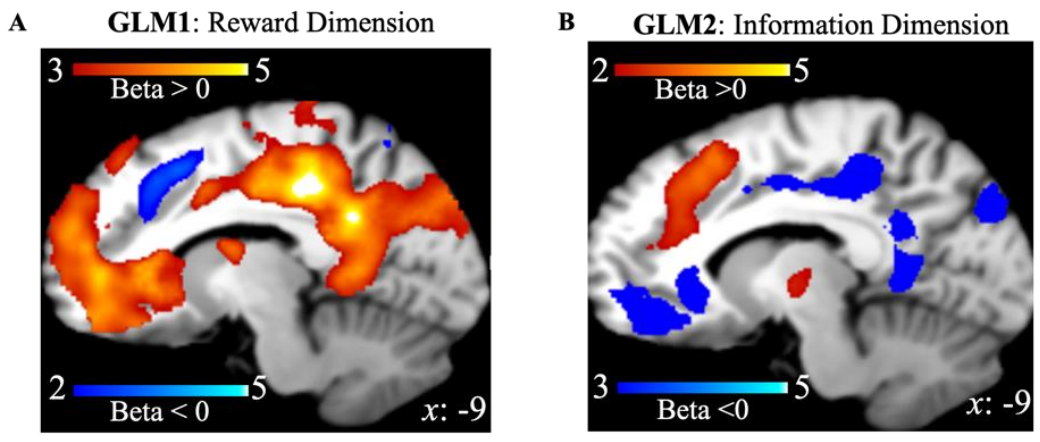

C

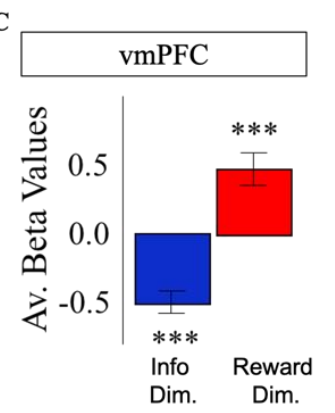

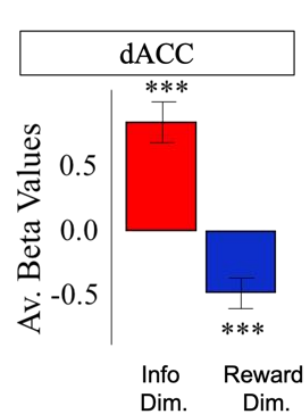

Dim. Rim.
D

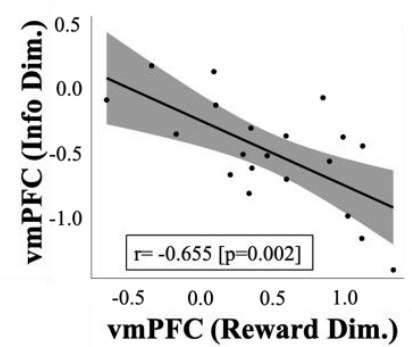

$\mathbf{E}$

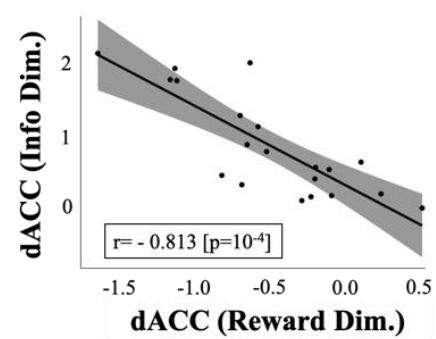

A) VMPFC positively correlated with model-based relative reward value for the selected option (in red), while dACC negatively correlated with it (in blue). B) DACC (in red) positively correlated with model-based information gain, while vmPFC negatively correlated with it (in blue). Activity scale represents z-score. Averaged BOLD beta estimates (C) for each ROI revealed a symmetrically-opposed pattern of activity in both Information and Reward dimensions, in line with model predictions when not controlling for potential correlations (Figure 1). BOLD signal estimates for Information Gain and RelReward Value were negatively correlated across subjects for both (D) vmPFC and (E) dACC ROIs.

203 In this section, we repeat our analyses while controlling for possible correlations between information and 204 reward that may underlie our results for GLMs $1 \& 2$. We investigated the effect of RelReward after 
controlling for Information Gain (GLM3), and the effect of Information Gain after controlling for RelReward (GLM4; Methods). Activity in vmPFC remained positively correlated with RelReward (FWE $p<0.001$, voxel extent $=1655$, peak voxel coordinates $(6,46,-2), t(19)=6.56$; Figure 4A) after controlling for Information Gain in GLM3. In contrast, whereas RelReward was negatively correlated with dACC activity in GLM1, no significant cluster was observed after the removing variance associated with

210 Information Gain in GLM3 as predicted by our model simulations (Figure 1H). Similarly, after controlling

211 for the effects of RelReward in GLM4, we observed significant activity in dACC positively correlated with 212 Information Gain (FWE $p<0.001$, voxel extent $=764$, peak voxel coordinates $(10,24,58), t(19)=5.89$;

213 Figure 4B), while we found no correlated activity in vmPFC as was observed in GLM2. A similar pattern 214 of activity was also observed when entering expected reward values, instead of their relative estimates, into 215 GLM4 (Supplement).

216 Correlations across subjects between the beta estimates for Information Gain (after controlling for 217 RelReward; GLM4) and RelReward (after controlling for Information Gain; GLM3) additionally suggest 218 that activity in vmPFC is specifically related to the relative reward value of the chosen deck (Figure 4C) 219 while activity in dACC is specifically related to the information to be gained from the chosen deck (Figure 220 4D) ${ }^{43}$. Directly contrasting the beta estimates for Information and RelReward in both clusters reveals an 221 asymmetrical pattern of activity in the two dimensions (Figure 4E). These results were replicated after 222 contrasting GLM3 and GLM4 using a paired-t-test (GLM3>GLM4: vmPFC (FWE $p<0.001$, voxel extent $223=467$, peak voxel coordinates $(-4,52,16), \mathrm{t}(19)=5.59)$; GLM4>GLM3: dACC (FWE $p<0.001$, voxel 224 extent $=833$, peak voxel coordinates $(10,24,46), t(19)=5.70)$; Supplement $)$.

225 To directly test our hypothesis that symmetric opposition between dACC and vmPFC is the product of 226 confounded reward and information signals, we conducted a 3-way ANOVA with ROI (dACC, vmpFC), 227 Value Type (Information Gain, RelReward), Analysis type (confounded\{GLM1\&2\}, no-confounded $228\{$ GLM3\&4\}) and we tested the 3-way interaction term. If independent reward and information value signals 229 are encoded in the brain, the two-way interaction (ROI x Value Type) should be significantly modulated 230 by the type of analysis adopted. Results showed a significant 3-way interaction $\mathrm{F}(1,19)=19.74, \mathrm{p}=0.0003$.

231 Altogether these findings suggest an independent evaluation of value and the coexistence of two 232 independent value systems for reward and information in human PFC. 
Figure 4. Independent value systems for reward and information in PFC

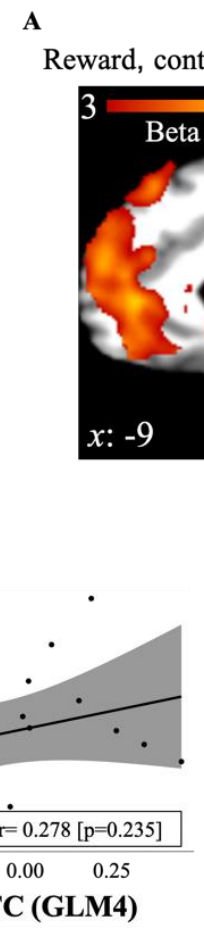

C

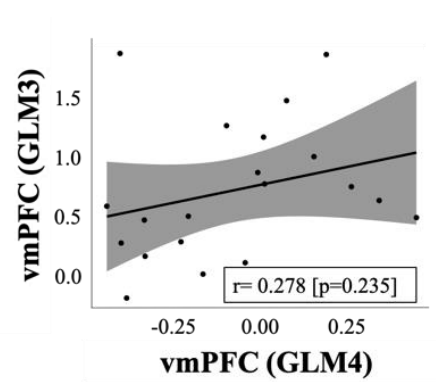

GLM3:

lling for Information Gain

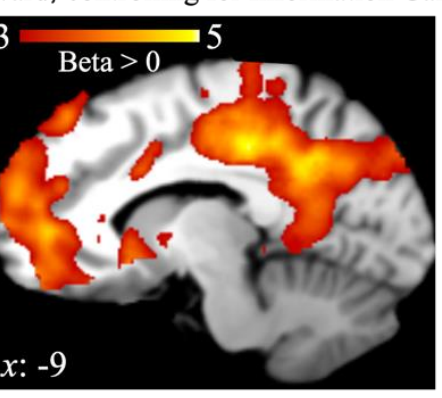

D

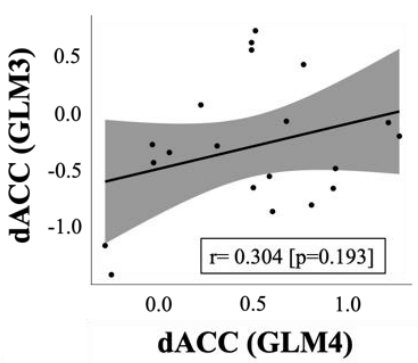

B Information Gain, controlling for Reward

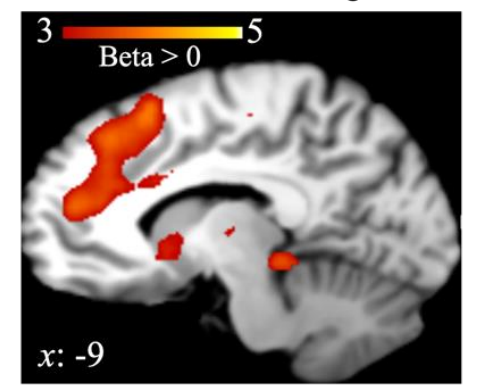

$\mathbf{E}$

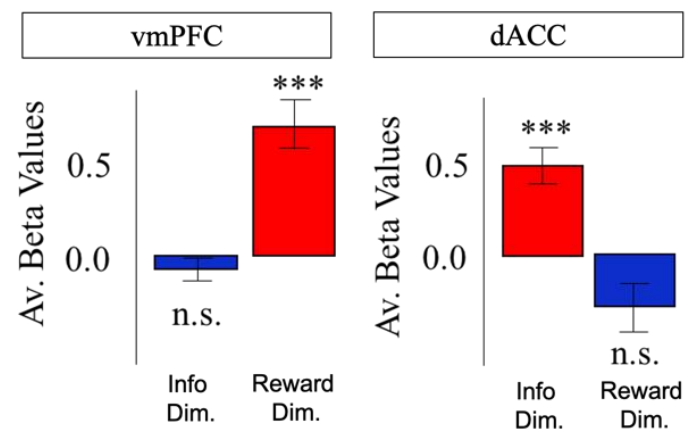

A) After controlling for information effects (GLM3), vmPFC activity (in red) positively correlated with model-based relative reward value (RelReward), while no correlations were observed for dACC. (B) After controlling for reward effects (GLM4), dACC activity (in red) positively correlated with model-based information gain (Information Gain), while no correlation was observed for vmPFC. The correlation of BOLD signal estimates between RelReward and Information Gain was no longer observed in either (C) vmPFC nor (D) dACC, and (E) comparison of average BOLD beta values confirms that effects of Information Gain are only observed in dACC, while those of RelReward are observed in vmPFC. "Info Dim" corresponds to the ROIs extracted from GLM4, while "Reward Dim" to the ROIs extracted from GLM3.

\section{Activity in dACC signals the non-instrumental value of information}

244 In the previous section, we showed that the value of information was independently encoded in dACC.

245 However, in our task different motives may drive participants to search for information ${ }^{44}$. Information can

246 be sought for its usefulness (i.e., instrumental utility): the acquired information can help with the goal of 247 maximizing points at the end of each game. Alternatively, information can be searched for its non248 instrumental value including novelty, curiosity or uncertainty reduction. Here, we tested whether the value 249 of information independently encoded in AACC relates to the instrumental value of information, to its non250 instrumental value or to both. 
251 We computed the instrumental value of information (Instrumental Information) by implementing a 252 Bayesian learner and estimating the Bayes optimal long-term value for the chosen option (Methods) on the

253 first free-choice trial. We first entered Instrumental Information and Information Gain in a mixed logistic 254 regression predicting first free choices with Instrumental Information and Information Gain as fixed effects, 255 subjects as random intercepts and 0+ Instrumental Information+ Information Gain | subjects as random 256 slope. Choices equal 1 when choosing most informative options, and 0 when choosing options selected 4 257 times during the forced-choice task. We found a positive effect of Information Gain (beta coefficient = $25872.07 \pm 16.91(\mathrm{SE}), \mathrm{z}=4.26, p=10^{-5}$ ) and a negative effect of Instrumental Information (beta coefficient $259=-2.25 \pm 0.464(\mathrm{SE}), \mathrm{z}=-4.85, p<10^{-6}$ ) on most informative choices. The percentage of trials in which 260 most informative choices had positive Instrumental Information was $~ 22 \%$ suggesting that in most of the 261 trials informative options were not selected based on instrumental utility.

262 We then entered Instrumental Information and Information Gain as parametric modulators into two 263 independent GLMs. We investigated the effects of Information Gain after controlling for Instrumental 264 Information (GLM5) and the effects of Instrumental Information after controlling for Information Gain 265 (GLM6; Methods). Activity in dACC positively correlated with Information Gain after controlling for 266 Instrumental Information in GLM5 (Figure 5A; FWE $p<0.001$, voxel extent $=1750$, peak voxel 267 coordinates $(10,26,56), t(19)=8.19)$. Activity in vmPFC was instead positively correlated with 268 Instrumental Information after controlling for Information Gain in GLM6 (Figure 5B; FWE $p<0.001$, 269 voxel extent $=557$, peak voxel coordinates $(20,20,-10), t(19)=5.62)$. These results suggest that activity 270 in dACC was strictly related to the non-instrumental value of information, while activity associated with 271 the instrumental value of information was expressed in reward regions consistent with ${ }^{45}$.

\section{Information value and choice difficulty}

273 Activity in dACC has been often associated with task difficulty ${ }^{28}$ and conflict ${ }^{46}$. Trials with greater levels 274 of choice difficulty or conflict may lead to prolonged reaction times, and AACC activity may index time on 275 task ${ }^{47}$ rather than task-related decision variables. In order to rule out the possibility that dACC activity, 276 associated with information value in our task, might instead be driven by time on task, we correlated the 277 standardized estimates of Information Gain with choice reaction times on the first free choice trials. The 278 correlation was run for each subject and correlation coefficients were tested against zeros using a Wilcoxon 279 Signed Test. Overall, correlation coefficients were not significantly different from zero (mean $\mathrm{r}=0.031$, $280 \mathrm{SE}=0.024 ; \mathrm{Z}=145 ; \mathrm{p}=0.1429)$ suggesting that pursuing an option with higher or lower information gain 
was not associated with higher or lower choice reaction times as predicted by a choice difficulty or conflict account of dACC function.

\section{dACC activity does not encode switching strategy}

A possible explanation for dACC activity associated with the non-instrumental value of information is that dACC encodes exploration or switching to alternative options away from a default choice. However, in our task the frequency of choosing the most informative option (i.e., exploration) was higher than the frequency of choosing the two other alternatives (in unequal condition, mean $=64.6 \%, \mathrm{SD}=18 \%$ ). Therefore, it is possible that in our task the default strategy was selecting the most informative options, and the switch strategy was selecting less informative (but potentially more rewarding) options. If this is correct, regions associated with exploration or switching strategy (e.g., frontopolar cortex ${ }^{36}$ ) should be activated when participants select a non-default option. We conducted a one sample t-test on the beta weights estimated for GLM7 which consists of a regressor modelling choice onset associated with the most informative options (Default), and another regressor modelling choice onset associated with the other two options (Switch).

294 Activity in frontopolar cortex was positively correlated with Switch (Switch-Default; Figure 5C; FWE $p$

$295=0.019$, voxel extent $=148$, peak voxel coordinates $(-18,52,6), t(19)=5.71)$. This suggests that adopting 296 a switching strategy in our task was associated with not choosing the most informative options, and not 297 with choosing highly informative options.

\section{Reward and information signal combine in the striatum}

299 While distinct brain regions independently encode values across different dimensions of the chosen option,

300 these values appear to converge at the level of the basal ganglia. In a final analysis (GLM8), we entered 301 choice probabilities derived from the RL model (where reward and information combine into a common 302 option value; eq. 4) as a single parametric modulator, and we observed positively-correlated activity in 303 bilateral ventral putamen (striatum region; right: FWE $p<0.01$, voxel extent $=238$, peak voxel coordinates $304(22,16,-6), t(19)=5.59)$; left: FWE $p<0.01$, voxel extent $=583$, peak voxel coordinates $(-26,8,-10)$, $305 \mathrm{t}(19)=5.89$; Figure 4D). Additionally, ventral putamen overlaps with voxels passing a threshold of $\mathrm{p}<$ 3060.001 for effects of both RelReward and Information Gain from GLMs 3 \& 4 (Figure 4E). 
$\mathbf{A}$

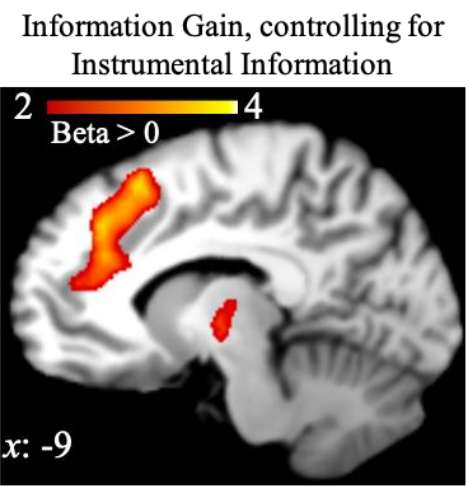

B Instrumental Information, controlling for Information Gain

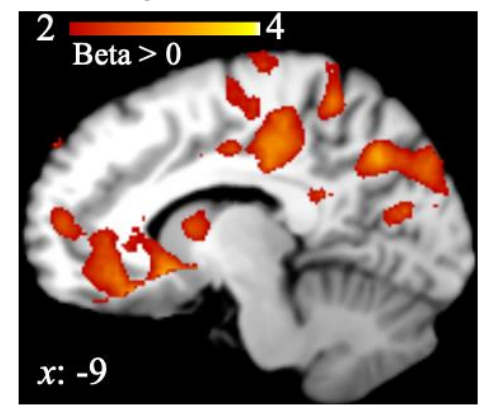

C

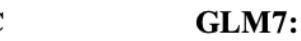

Switch-Default

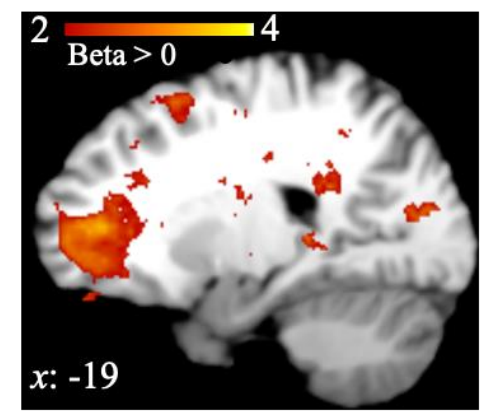

D

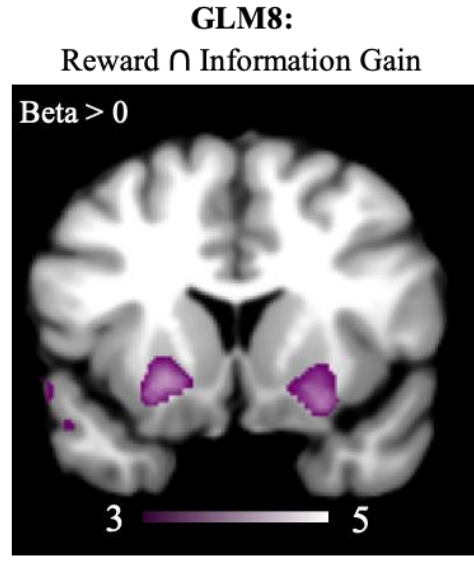

$\mathbf{E}$

$$
\begin{gathered}
\text { Reward U Information Gain } \\
\text { overlapping regions }
\end{gathered}
$$

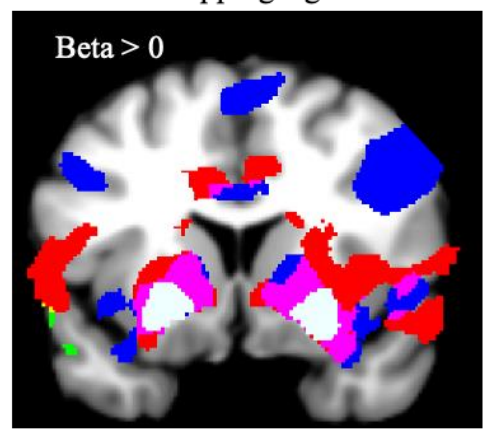

$$
y=11, z=-5
$$

A) Activity in dACC correlated with Information Gain after controlling for the instrumental value of information. B) Activity in vmPFC correlated with the instrumental value of information after controlling for Information Gain. C) Activity in frontopolar region correlated with Switch (not choosing the most informative options)- Default strategy (choosing most informative options). G) Activity in the ventral putamen (striatum region) correlated with response probabilities derived from the RL model and $\mathbf{H}$ ) both RelReward and Information Gain overlap in the striatum region (in white). Activity scale represents z-score. 


\section{DISCUSSION}

319 Decisions are often influenced by both potential reward and information gains associated with options 320 available in the environment ${ }^{25}$. Here, we present evidence for dedicated and independent value systems for 321 such decision variables in the human PFC. When correlations between reward and information were taken 322 into account, we found that dACC and vmPFC distinctly encoded information value and relative reward 323 value of the chosen option, respectively. These value signals were then combined in subcortical regions in 324 order to implement choices. These findings are direct empirical evidence for a dedicated information value 325 system in human PFC, independent of reward value. Our finding is in line with a view of human PFC as an 326 optimizer of independent value signals ${ }^{10,12,48}$.

327 Our main finding that $\mathrm{AACC}$ and vmPFC distinctly encode information gain and relative reward supports theoretical accounts such as active inference ${ }^{26} 49$ and certain RL models (e.g., upper confidence bound ${ }^{47}$ ) which predict independent computations in the brain for information value (epistemic value) and reward value (extrinsic value). Consistent with our findings, the activity of single neurons in the monkey orbitofrontal cortex independently and orthogonally reflects the output of the two value systems ${ }^{50}$.

332 Therefore, our results may highlight a general coding scheme that the brain adopts during decision-making 333 evaluation.

334 Our finding that activity in dACC positively correlates with the information value of the chosen option 335 suggests the existence of a dedicated system for information in the human PFC independent of the reward 336 value system. Our control analysis also suggests that dACC encodes the non-instrumental value of 337 information, while the instrumental value of information was expressed in reward regions as previously 338 suggested ${ }^{45}$. Our results are in line with recent findings in monkey literature that identified a population of 339 neurons in dACC which selectively encodes the non-instrumental value of information ${ }^{51}$. Additionally, our 340 results are consistent with computational models of PFC which predict that ACC activity can be primarily 341 explained as indexing prospective information about an option independent of reward value ${ }^{37,52,53}$. DACC 342 has often been associated with conflict ${ }^{46}$ and uncertainty ${ }^{41}$, and recent findings suggest that activity in the 343 region corresponds to unsigned prediction errors, or "surprise" 54 . Our results enhance this perspective by 344 showing that the activity observed in dACC during decision-making can be explained as representing the 345 subjective representation of decision variables (i.e., information value signal) elicited in uncertain or novel 346 environments. It is worth highlighting that other regions might be involved in processing information347 related components of the value signal not elicited by our task. In particular, orbitofrontal cortex signals the 348 opportunity to receive knowledge vs. ignorance ${ }^{18}$ and rostrolateral PFC signals the changes in relative 
uncertainty associated with the exploration of novel and uncertain environments ${ }^{55}$. Neural recordings in monkeys also showed an interconnected cortico-basal ganglia network which resolve uncertainty during information-seeking ${ }^{51}$. Taken together, these findings highlight an intricate and dedicated network for information, independent of reward. Further research is therefore necessary to map the information network in the human brain and understand to what extent this network relies on neural computations so far associated with reward processing (e.g, dopaminergic modulations ${ }^{56}{ }^{57}$ ).

Our finding that vmpFC positively correlates with the relative reward value of the chosen option agrees with previous research that identifies vmPFC as a region involved in value computation and reward processing ${ }^{58}$. VmPFC appears not only to code reward-related signals ${ }^{59} 60,61$ but to specifically encode the relative reward value of the chosen option ${ }^{62}$, in line with the results of our study. We also observed clusters in posterior cingulate cortex which were positively correlated with the relative reward value of the chosen option in a similar fashion as observed for vmPFC, suggesting a role of posterior cingulate in reward processing and exploitative behaviors as previously reported in monkey studies ${ }^{6364}$.

362 Our results further suggest that these independent value systems interact in the striatum, consistent with its 363 hypothesized role in representing expected policies ${ }^{65}$. The convergence of reward and information signal 364 in the striatum region is also consistent with the identification of basal ganglia as a core mechanism that 365 supports stimulus-response associations in guiding actions ${ }^{66}$ as well as recent findings demonstrating 366 distinct corticostriatal connectivity for affective and informative properties of a reward signal ${ }^{67}$. 367 Furthermore, our results are in line with recent evidence on multidimensional value encoding, as opposed 368 to "pure" value encoding, in the striatum ${ }^{689} 70$. Moreover, activity in this region was computed from the 369 softmax probability derived from our RL model, consistent with previous modeling work that identified the 370 basal ganglia as the output of the probability distribution expressed by the softmax function ${ }^{71}$.

371 In addition to dACC, we observe activity in additional regions of the cognitive control network which 372 correlate with the information value signal, including bilateral anterior insula cortex and dorsolateral PFC 373 (dlPFC). Activity in these regions is frequently observed in conjunction with dACC activity. Although 374 activity in these additional regions correlates with information value, it is unclear whether they, like dACC,

375 represent information value per se, or instead may represent variables that correlate with information value 376 but were not controlled for in this experiment, e.g., context uncertainty ${ }^{53}$. Additional work is needed to 377 determine the unique contributions of these regions in signaling information value. 
At the same time, our results suggest that the symmetrical opposition between dACC and vmPFC frequently observed in the literature 27,30 31 32,33 34,35 can emerge as results of correlated variables. The results of our study are in line with O'Doherty ${ }^{72}$ who warned of the possibility that, in most neuroeconomics and decision neuroscience studies activity identified as a value signal might instead capture informational signaling of an outcome or particular hidden structure of a decision problem. Recent work has emphasized ecologicallyvalid tasks for investigating behavior and brain function; while it is critical to characterize the function of

384 brain structures in terms of the behaviors they evolved to support, increased task realism frequently entails

385 a loss of control over experimental variables. Furthermore, we acknowledge that this confound may not

386 emerge in every decision-making studies (e.g., preference-based choice). In those studies, the confound is

387 reflected in subjects' previous experiences (e.g., the expression of a preference for one type of food over

388 another are consistent, and therefore the subject reliably selects that food type over others on a regular

389 basis). The control of this confound is even more tricky as it is "baked in" by prior experiences rather than

390 learned over the course of an experimental session. Additionally, our results suggest that other decision

391 dimensions involved in most decision-making tasks (e.g., effort and motivation, cost, affective valence, or

392 social interaction) may also be confounded in the same manner. Indeed, symmetrical opposition between

393 dACC and vmPFC has been reported for a wide range of contexts involving decision variables such as

394 effort, delay, and affective valence (Table S1). Our findings therefore suggest caution is needed when

395 interpreting findings from such tasks.

396 Taken together, by showing the existence of independent value systems in the human PFC, this study 397 provides the first empirical evidence in support of a theoretical work aimed at developing a unifying 398 framework for interpreting brain functions. Additionally, this study individuates a dedicated value system 399 for information, independent of reward value. Overall, our results suggest a new perspective on how to look 400 at decision-making processes in the human brain under realistic scenarios, with potential implications for 401 the interpretation of PFC activity in both healthy and clinical populations. 


\section{METHODS}

\section{Participants}

405 Twenty-one right-handed, neurologically healthy young adults were recruited for this study (12 women; aged $40619-29$ years, mean age $=23.24)$. Of these, one participant was excluded from the analysis due to problems 407 in the registration of the structural $\mathrm{T}_{1}$ weighted MPRAGE sequence. The sample size was based on previous 408 studies e.g., ${ }^{30} 33$ 28 . Participants also presented normal color vision and absence of psychoactive treatment. 409 The entire group belonged to the Belgian Flemish-speaking community. The experiment was approved by 410 the Ethical Committee of the Ghent University Hospital and conducted according to the Declaration of 411 Helsinki. Informed consent was obtained from all participants prior to the experiment.

\section{Procedure}

413 Participants performed a gambling-task where on each trial choices were made among three decks of cards

$414{ }^{25}$ (Figure 2). The gambling-task consisted of 128 games. Each game contains two phases: a forced-choice 415 task where participants selected options highlighted by the computer for 6 consecutive trials, and a free416 choice task where participants produced their own choices in order to maximize the total gain obtained at 417 the end of the experiment (from 1 to 6 trials, exponentially inversely distributed such that subjects were 418 most frequently allowed to make 6 free choices). The free choice trial length or horizon was not cued to 419 participants. Therefore, on each game participants were not aware of the free choice horizon. We have 420 already shown that the choice horizon does not affect participants' choices on this task ${ }^{25} 73$. In the forced421 choice task, participants were forced to either choose each deck 2 times (equal information condition), or 422 to choose one deck 4 times, another deck 2 times, and 0 times for the remaining deck (unequal information 423 condition). By using this two phase-task, Wilson et al. showed that the difference in the number of time 424 each option is sampled and the differences in the mean reward is orthogonalized ${ }^{20}$ (i.e., options associated 425 with the lowest amount of information were least associated with experienced reward values). In other 426 words, the use of the forced-choice task allows to orthogonalize available information and reward delivered 427 to participants in the first free choice trial. For this reason, the focus of our fMRI analyses is on the first 428 free-choice of each game (resulting in 128 trials for the fMRI analysis). We adopted, however, trial-by-trial 429 fMRI analyses to have a better estimate of neural activity over the overall performance. Therefore, we 430 treated equal information condition and unequal information condition altogether.

431 On each trial, the payoff was generated from a Gaussian distribution with a generative mean between 10 and 43270 points and standard deviation of 8 points. The generative mean for each deck was set to a base value of 
433 either 30 or 50 points and adjusted independently by $+/-0,4,12$, or 20 points with equal probability, to avoid 434 the possibility that participants might be able to discern the generative mean for a deck after a single 435 observation. The generative mean for each option was stable within a game, but varied across games.

436 Participants' payoff on each trial ranged between 1 and 100 points and the total number of points was summed 437 and converted into a monetary payoff at the end of the experimental session ( 0.01 euros every 60 points). 438 Participants were told that during the forced-choice task, they may sample options at different rates, and that 439 the decks of cards did not change during each game, but were replaced by new decks at the beginning of each 440 new game. However, they were not informed of the details of the reward manipulation or of the underlying 441 generative distribution adopted during the experiment. Participants underwent a training session outside the 442 scanner in order to make the task structure familiar to them.

443 The forced-choice task lasted about $8 \mathrm{sec}$ and was followed by a blank screen, for a variable jittered time 444 window $(1 \mathrm{sec}-7 \mathrm{sec})$. The temporal jitter allows to obtain neuroimaging data at the onset of the first-free 445 choice trial and right before the option was selected (decision window). After participants performed the 446 first free-choice trial, a blank screen was again presented for a variable jittered time window (1 sec - $6 \mathrm{sec})$ 447 before the feedback, indicating the number of points earned, was given for $0.5 \mathrm{sec}$ and another blank screen 448 was shown to them for a variable jittered time window. As the first free-choice trial was the main trial of 449 interest for the fMRI analysis, subsequent free-choice trials were not jittered.

\section{$450 \quad$ Image acquisition}

451 Data was acquired using a 3T Magnetom Trio MRI scanner (Siemens), with a 32-channel radio-frequency 452 head coil. In an initial scanning sequence, a structural $\mathrm{T}_{1}$ weighted MPRAGE sequence was collected (176 453 high-resolution slices, $\mathrm{TR}=1550 \mathrm{~ms}, \mathrm{TE}=2.39$, slice thickness $=0.9 \mathrm{~mm}$, voxel size $=0.9 \times 0.9 \times 0.9 \mathrm{~mm}$, $454 \mathrm{FoV}=220 \mathrm{~mm}$, flip angle $=9^{\circ}$ ). During the behavioral task, functional images were acquired using a $\mathrm{T}_{2}{ }^{*}$ 455 weighted EPI sequence ( 33 slices per volume, $\mathrm{TR}=2000 \mathrm{~ms}$, TE $=30 \mathrm{~ms}$, no inter-slice gap, voxel size = $4563 \times 3 \times 3 \mathrm{~mm}, \mathrm{FoV}=192 \mathrm{~mm}$, flip angle $=80^{\circ}$ ). On average 1500 volumes per participant were collected 457 during the entire task. The task lasted approximately $1 \mathrm{~h}$ split in 4 runs of about 15 minutes each.

\section{Behavioral Analysis}

\section{Expected reward value and information value}

460 To estimate participants' expected reward value and information value, we adopted a previously 461 implemented version of a reinforcement learning model that learns reward values and information gained 
462 about each deck during previous experience - the gamma-knowledge Reinforcement Learning model $463\left(\mathrm{gkRL} ;{ }^{25,73}\right)$. This model was already validated for this task and it was better able to explain participants' 464 behavior compared to other RL models ${ }^{4}$.

465 Expected reward values were learned by gkRL adopting on each trial a simple $\delta$ learning rule ${ }^{74}$ :

$$
Q_{t+1, j}(c)=Q_{t, j}(c)+\alpha \times \delta_{t, j}(1)
$$

467 where $Q_{t, j}(c)$ is the expected reward value for deck $c$ (= Left, Central or Right) at trial $t$ and game $j$ 468 and $\delta_{t, j}=R_{t, j}(c)-Q_{t, j}(c)$ is the prediction error, which quantifies the discrepancy between the previous 469 predicted reward values and the actual outcome obtained at trial $t$ and game $j$.

470 Information was computed as follows:

$$
I_{t, j}(c)=\left(\sum_{1}^{t} i_{t, j}(c)\right)^{\gamma}
$$

$$
\text { where, } i_{t, j}(c)=\left\{\begin{array}{l}
0, \text { choice } \neq c \\
1, \text { choice }=c
\end{array}\right.
$$

$473 I_{t, j}(c)$, is the amount of information associated with the deck $c$ at trial $t$ and game j. $I_{t, j}(c)$, is computed by 474 including an exponential term $\gamma$ that defines the degree of non-linearity in the amount of observations 475 obtained from options after each observation. $\gamma$ is constrained to be $>0$. Each time deck c is selected, $i_{t, j}(c)$ 476 takes value of 1 , and 0 otherwise. On each trial, the new value of $i_{t, j}(c)$ is summed to the previous $477 i_{t-1,1: j}(c)$ estimate and the resulting value is elevated to $\gamma$, resulting in $I_{t, j}(c)$.

478 Before selecting the appropriate option, gkRL subtracts the information gained $I_{t, j}(c)$ from the expected 479 reward value $Q_{t+1, j}(c)$ :

$$
V_{t, j}(c)=Q_{t+1, j}(c)+\left(-I_{t, j}(c) * \omega\right)(3)
$$


$V_{t, j}(c)$ is the final value associated with deck $c$. Here, information accumulated during the past trials scales

482 values $V_{t, j}(c)$ so that increasing the number of observations of one option decreases its final value. The

483 scaling is controlled by $\omega$ which defines the degree new information is integrated in the choice value.

In order to generate choice probabilities based on expected reward and information values (i.e., final choice value), the model uses a softmax choice function ${ }^{75}$. The softmax rule is expressed as:

$$
P\left(c / V_{t, j}\left(c_{i}\right)\right)=\frac{\exp \left(\beta \times V_{t, j}(c)\right)}{\sum_{i} \exp \left(\exp \beta \times V_{t, j}\left(c_{i}\right)\right)}
$$

where $\beta$ is the inverse temperature that determines the degree to which choices are directed toward the highest rewarded option. By minimizing the negative log likelihood of $P\left(c / V_{t, j}\left(c_{i}\right)\right)$ model parameters $\alpha$, $\beta$, and $\omega, \gamma$, were estimated for participants' choices made during the first free-choice trials. The fitting procedure was performed using MATLAB and Statistics Toolbox Release 2020a function fminsearch and its accuracy tested using parameter recovery analysis (Supplement). Model parameters were then used to compute the value of $Q_{t+1, j}(c)$ and $I_{t, j}(c)$ for each participant. The results of this fit are reported in the table S2.

In order to approximate the instrumental utility of options in our task, we turn to Bayesian modeling. In the simplest case, a decision-maker's choice when confronted with multiple options depends on its beliefs about the relative values of those options. This requires the decision-maker to estimate, based on prior experience, relevant parameters such as the mean value and variance of each option. On one hand, the mean and variance of an option can be estimated through direct experience with that option through repeated sampling. However, subjects may also estimate long-term reward contingencies as well: even if an option has a specific mean reward during one game in our task, subjects may learn an estimate of the range of rewards that options can have even before sampling from any option. Similarly, although subjects may

503 learn an estimate of the variance for a specific option during the forced choice period, over many games subjects may learn that options in general have a variance around a specific value.

To model this, we developed a Bayesian learner that estimates, during each game, the probability distribution over reward and variance for each specific option in that game, and, over the entire experiment, estimates the global distribution over mean reward and variance based on observed rewards from all options. A learner's belief about an option can be modeled as a joint probability distribution over likely 
509 values for the mean reward (mu) and standard deviation (sigma). In order to reduce computational demands

510 when conducting forward searches with the model (see below), sigma values were modeled as the integers

511 from 1 to 25 and the range of rewards was modeled in 10 point increments from 5 to 95 . Prior to any

512 exposure to the task, the probability distributions over mu and sigma were initialized as a uniform

513 distribution.

514 To model training received by each subject prior to participation in the experiment, the Bayesian learner

515 was simulated on forced choices from 10 random games generated from the same routine used to generate

516 trials during the experiment. After each choice was displayed, the global probability distribution over mu

517 and sigma was adjusted using Bayesian updating:

$$
P(\mu, \sigma \mid R) \propto P(\mu, \sigma) * N(R \mid \mu, \sigma)(5)
$$

where $\mathrm{N}()$ is the probability of observing a reward for a normal distribution with a given mean and variance.

520 Following the initial training period, the model performed the experiment using games experienced by the

521 subjects themselves, i.e., during the forced choice period, the model made the same choices and observed

522 the same point values seen during the experiment. To model option-specific estimates, the model

523 maintained three probability distributions over $\mu$ and $\sigma$ corresponding to each option, essentially a local

524 instantiation of the global probability distribution described above. The option-specific distributions were

525 reset to the global prior distribution before each new game, and were updated only after an outcome was

526 observed for that option using the same updating rule described in eq. 5.

527 The Bayesian learner described above learns to estimate the probability distribution over the mean and 528 variance for each option during the forced-choice component of the experiment. If the learner's only 529 concern in the free-choice phase is to maximize reward for the next choice, it would select the option with 530 the highest expected value. However, in our task, subjects are instructed to maximize their total return for 531 a variable number of trials with the same set of options. In some circumstances, it is better to select from 532 under-sampled decks that may ultimately have a higher value than the current best estimate.

533 To model this, we implemented a forward tree search algorithm ${ }^{76,77}$ which considers all choices and 534 possible outcomes ("states") reachable from the current state, updates the posterior probability distribution 535 for each subsequent state as described above, and repeats this from the new state until a fixed number of 
536 steps have been searched. By conducting an exhaustive tree search to a given search depth, it is possible to

537 determine the Bayes optimal choice at the first free-choice trial in our experiment.

538 In practice, however, it is usually unfeasible to perform an exhaustive search for any but the simplest 539 applications (limited branching factor, limited horizon). In our experiment, the outcome of a choice was an 540 integer from 1 to 100 (\# of points), and the model could select from 3 different options, yielding a branching 541 factor of 300. The maximum number of free-choice trials available on a given game was 6 , meaning that a 542 full search would consider $3^{\wedge} 8$ possible states at the terminal leaves of the tree. In order to reduce the time 543 needed to perform a forward tree search of depth 6, we applied a coarse discretization to the possible values

544 of mu and sigma (i.e., sigma values were modeled as the integers from 1 to 25 and the range of rewards 545 was modeled in 10 point increments from 5 to 95). We additionally pruned the search tree during runtime 546 such that any branch that had a probability less than 0.001 of being observed was removed from further 547 consideration.

548 The value of a state was modeled as the number of points received for reaching that state, plus the maximum 549 expected value of subsequent states that could be reached. Thus, the value of leaf states was simply the 550 expected value of the probability distribution over means (numerically integrated over sigma), while the 551 value of the preceding state was that state's value plus the maximum expected value of possible leaf states:

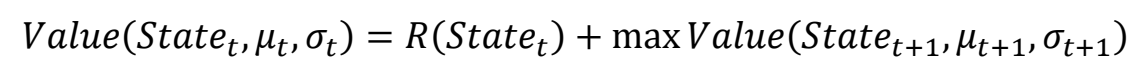

553 Recursively applying eq. 6 from the leaf states to the first free-choice trial allows us to approximate the 554 Bayes optimal long-term value for each option.

555 This algorithm was run for each game experienced by a subject during the experiment in order to derive the 556 expected instrumental value (Instrumental Information) for each option following the forced-choice trials 557 specific to that game.

\section{$558 \quad$ fMRI analysis}

559 The first 4 volumes of each functional run were discarded to allow for steady-state magnetization. The data 560 were preprocessed with SPM12 (Wellcome Department of Imaging Neuroscience, Institute of Neurology, 561 London, UK). Functional images were motion corrected (by realigning to the first image of the run). The 562 structural $\mathrm{T}_{1}$ image was coregistered to the functional mean image for normalization purposes. Functional 
images normalized to a standardized (MNI) template (Montreal Neurological Institute) and spatially smoothed with a Gaussian kernel of 8 mm full width half maximum (FWHM).

All the fMRI analyses focus on the time window associated with the onset of the first free-trials prior the choice was actually made (see Procedure). The rationale for our model-based analysis of fMRI data is as follows (Table S3). First, in order to link participants' behavior with neural activity, GLM0 was created with a regressor modelling choice onset associated with highest rewarded options (Highest Reward), and another regressor modelling choice onset associated with lower rewarded options (Lower Reward). Activity related to Highest Reward was then subtracted from the activity associated with Lower Reward (giving a value 1 and -1 respectively) at the second level. Next, in order to identify regions with activity related to reward and information, we computed the relative value of the chosen deck (RelReward) and the (negative) value of gkrl model-derived information gained from the chosen option (Information Gain). RelReward was computed by subtracting the average expected reward values for the unchosen decks from the expected reward values of the chosen deck $c$ from the gkrl model $\left(Q_{t+1, j}^{R}(c=1)=Q_{t+1, j}(c=1)-\right.$ mean $\left(Q_{t+1, j}(c=2), Q_{t+1, j}(c=3)\right)$. We adopted a standard computation of relative reward values ${ }^{28}$. It has already been shown that vmPFC represents reward values following the above computation. However, we compare these computations with expected reward values or additional covariates (e.g., chosen-second best option, average of the chosen decks, etc. Supplement). Information Gain was computed as $-I_{t, j}(c)$. The negative value $I_{t, j}(c)$ relates to the information to be gained about each deck by participants. We have already shown that humans represent information value as computed by our model compared to alternative computations when performing the behavioral task adopted in this study ${ }^{25}$. Next, we entered RelReward and Information Gain as parametric modulators into GLMS with a single regressor modeling the onset of the first free-choice trial as a 0 duration stick function. In GLM1, RelReward was included a single parametric modulator. In GLM2, Information Gain was included as a single parametric modulator. In GLM3, two parametric modulators were included in the order: Information Gain, RelReward. In GLM4, the same two parametric modulators were included, with the order reversed i.e., RelReward, Information Gain. The intent of GLMs $3 \& 4$ was to allow us to investigate the effects of the $2^{\text {nd }}$ parametric modulator after accounting for variance that can be explained by the $1^{\text {st }}$ parametric modulator. In SPM12, this is accomplished by enabling modulator orthogonalization (Wellcome Department of Imaging 591 Neuroscience, Institute of Neurology, London, UK). To determine the regions associated with Reward and 592 Information Gain, beta weights for the first (single modulator GLMS) or second (two modulator GLMS) 593 parametric modulators were entered into a $2^{\text {nd }}$ level (random effects) paired-sample t-test. In order to 594 determine activity related to the combination of information and reward value, GLM8 was created with the 595 softmax probability of the chosen option $\left(P\left(c / V_{t, j}\left(c_{i}\right)\right)\right.$ modelling the onsets of first free-choices. 
596 Additional GLMs where then created for the control analyses: GLM1bis with expected reward value

597 (ExpReward) as single parametric modulator; GLM4bis with two parametric modulators ExpReward and

598 Information Gain; GLM5 and 6 with Instrumental Information and Information Gain as parametric

599 modulators; GLM7 which comprises of a regressor modelling choice onset associated with the most

600 informative options (Default), and another regressor modelling choice onset associated with the other two

601 options (Switch); GLM9 and 10 with ExpReward and RelReward as parametric modulators; and GLM11

602 where the maximum value of 3 decks (Max Value), the minimum value of the 3 decks (Min Value), the

603 reward value variation for the chosen option (Standard Deviation), the averaged value of the 3 decks

604 (Averaged Value), the value of the chosen option minus the value of the best second option (Chosen-

605 Second) and RelReward compete for variance.

606 Activity for these GLMs are reported either in the main text or in Table S4.

607 In order to denoise the fMRI signal, 24 nuisance motion regressors were added to the GLMs where the

608 standard realignment parameters were non-linearly expanded incorporating their temporal derivatives and

609 the corresponding squared regressors ${ }^{78}$. Furthermore, in GLMS with two parametric modulators, regressors

610 were standardized to avoid the possibility that parameter estimates were affected by different scaling of the

611 models' regressors alongside with the variance they might explain ${ }^{79}$. During the second level analyses, we

612 corrected for multiple comparisons in order to avoid the false positive risk ${ }^{80}$. We corrected at cluster level

613 using both FDR and FEW. Both corrections gave similar statistical results therefore we reported only FEW

614 corrections. 


\section{REFERENCES}

6171 Rangel, A., Camerer, C. \& Montague, P. R. A framework for studying the neurobiology

618 of value-based decision making. Nat Rev Neurosci 9, 545-556, doi:10.1038/nrn2357

619

6202 Doya, K. Modulators of decision making. Nat Neurosci 11, 410-416, doi:10.1038/nn2077

$621 \quad$ (2008).

6223 Montague, P. R., King-Casas, B. \& Cohen, J. D. Imaging valuation models in human choice. Annu Rev Neurosci 29, 417-448, doi:10.1146/annurev.neuro.29.051605.112903 (2006). Glimcher, P. W., Camerer, C., Fehr, E. \& Poldrack, R. A. Neuroeconimcs-decision-

4 Glimcher, P. W., Camerer, C., Fehr, E. \& Poldrack

5 Sutton, R. S. \& Barto, A. G. Reinforcement Learning: An introduction. (MIT Press, 1998).

6 Ellsberg, D. Risk, ambiguity, and the Savage axioms. Q. J. Econ 75, 643-669, doi:doi: $10.2307 / 1884324$ (1961).

7 Rosati, A. G., Stevens, J. R., Hare, B. \& Hauser, M. D. The evolutionary origins of human patience: temporal preferences in chimpanzees, bonobos, and human adults. Curr Biol 17, 1663-1668, doi:10.1016/j.cub.2007.08.033 (2007).

8 Botvinick, M. M., Huffstetler, S. \& McGuire, J. T. Effort discounting in human nucleus accumbens. Cogn Affect Behav Neurosci 9, 16-27, doi:10.3758/CABN.9.1.16 (2009). Rushworth, M. F., Kolling, N., Sallet, J. \& Mars, R. B. Valuation and decision-making in frontal cortex: one or many serial or parallel systems? Curr Opin Neurobiol 22, 946-955, doi:10.1016/j.conb.2012.04.011 (2012). inference, evidence accumulation, and the urn task. Neural Comput 27, 306-328, doi:10.1162/NECO_a_00699(2015).

12 Friston, K. Learning and inference in the brain. Neural Netw 16, 1325-1352, doi:10.1016/j.neunet.2003.06.005 (2003).

13 Kidd, C. \& Hayden, B. Y. The Psychology and Neuroscience of Curiosity. Neuron 88, 449-460, doi:10.1016/j.neuron.2015.09.010 (2015).

14 Bellemare, M. G. et al. Unifying count-based exploration and intrinsic motivation. Advances in Neural Information Processing Systems (2016).

15 Singh, S., Barto, A. G. \& Chentanez, N. Intrinsically motivated reinforcement learning. Adv. Neural Inform. Process. Syst. 17 (2005).

16 Chung, J. J., Lawrance, N. R. J. \& Sukkarieh, S. Learning to soar: Resource-constrained exploration in reinforcement learning. The international journal of robotics research $34,158-$ $172(2015)$.

17 Ecoffet, A., Huizinga, J., Lehman, J., Stanley, K. O. \& Clune, J. Go-Explore: a New Approach for Hard-Exploration Problems. arXiv:1901.10995 (2019).

18 Charpentier, C. J., Bromberg-Martin, E. S. \& Sharot, T. Valuation of knowledge and ignorance in mesolimbic reward circuitry. Proc Natl Acad Sci U S A 115, E7255-E7264, doi:10.1073/pnas.1800547115 (2018).

661

19 Kobayashi, K., Ravaioli, S., Baranes, A., Woodford, M. \& Gottlieb, J. Diverse motives for human curiosity. Nat Hum Behav 3, 587-595, doi:10.1038/s41562-019-0589-3 (2019). 
66220 Wilson, R. C., Geana, A., White, J. M., Ludvig, E. A. \& Cohen, J. D. Humans use directed and random exploration to solve the explore-exploit dilemma. Journal of experimental psychology. General 143, 2074-2081, doi:10.1037/a0038199 (2014). Wu, C. M., Schulz, E., Speekenbrink, M., Nelson, J. D. \& Meder, B. Generalization guides human exploration in vast decision spaces. Nat Hum Behav 2, 915-924, doi:10.1038/s41562-018-0467-4 (2018).

22 Martinelli, C., Rigoli, F., Averbeck, B. \& Shergill, S. S. The value of novelty in schizophrenia. Schizophr Res 192, 287-293, doi:10.1016/j.schres.2017.05.007 (2018).

23 Hildebrand-Saints, L. \& Weary, G. Depression and social information gathering. Personality and Social Psychology Bulletin 15, 150-160 (1989).

24 Cogliati Dezza, I., Noel, X., Cleeremans, A. \& Yu, A. J. What drive information-seeking in healthy and addicted behaviors. bioRxiv (2020).

25 Cogliati Dezza, I., Yu, A. J., Cleeremans, A. \& Alexander, W. Learning the value of information and reward over time when solving exploration-exploitation problems. Sci Rep 7, 16919, doi:10.1038/s41598-017-17237-w (2017).

26 Shenhav, A., Botvinick, M. M. \& Cohen, J. D. The expected value of control: an integrative theory of anterior cingulate cortex function. Neuron 79, 217-240, doi:10.1016/j.neuron.2013.07.007 (2013).

27 Shenhav, A., Straccia, M. A., Botvinick, M. M. \& Cohen, J. D. Dorsal anterior cingulate and ventromedial prefrontal cortex have inverse roles in both foraging and economic choice. Cogn Affect Behav Neurosci 16, 1127-1139, doi:10.3758/s13415-016-0458-8 (2016). engagement in a foraging context reflects choice difficulty, not foraging value. Nat Neurosci 17, 1249-1254, doi:10.1038/nn.3771 (2014).

29 Shenhav, A., Cohen, J. D. \& Botvinick, M. M. Dorsal anterior cingulate cortex and the value of control. Nat Neurosci 19, 1286-1291, doi:10.1038/nn.4384 (2016).

30 Kolling, N., Behrens, T. E., Mars, R. B. \& Rushworth, M. F. Neural mechanisms of foraging. Science 336, 95-98, doi:10.1126/science.1216930 (2012).

31 Kolling, N., Wittmann, M. \& Rushworth, M. F. S. Multiple neural mechanisms of decision making and their competition under changing risk pressure. Neuron 81, 11901202, doi:10.1016/j.neuron.2014.01.033 (2014).

32 Wittmann, M. K. et al. Predictive decision making driven by multiple time-linked reward representations in the anterior cingulate cortex. Nat Commun 7, 12327, doi:10.1038/ncomms12327 (2016).

33 Boorman, E. D., Rushworth, M. F. \& Behrens, T. E. Ventromedial prefrontal and anterior cingulate cortex adopt choice and default reference frames during sequential multialternative choice. J Neurosci 33, 2242-2253, doi:10.1523/JNEUROSCI.3022-12.2013 (2013).

34 Arulpragasam, A. R., Cooper, J. A., Nuutinen, M. R. \& Treadway, M. T. Corticoinsular circuits encode subjective value expectation and violation for effortful goal-directed behavior. Proc Natl Acad Sci U S A 115, E5233-E5242, doi:10.1073/pnas.1800444115 (2018). maximizing rewards: computational principles and neural correlates. J Neurosci 34, 15621-15630, doi:10.1523/JNEUROSCI.1350-14.2014 (2014). 
Daw, N. D., O'Doherty, J. P., Dayan, P., Seymour, B. \& Dolan, R. J. Cortical substrates for exploratory decisions in humans. Nature 441, 876-879, doi:10.1038/nature04766 (2006).

Behrens, T. E., Woolrich, M. W., Walton, M. E. \& Rushworth, M. F. Learning the value of information in an uncertain world. Nat Neurosci 10, 1214-1221, doi:10.1038/nn1954 (2007). Anterior Cingulate in Subjective Valuation of Prospective Effort. Cereb Cortex 29, 4277 4290, doi:10.1093/cercor/bhy310 (2019).

39 Marsh, A. A., Blair, K. S., Vythilingam, M., Busis, S. \& Blair, R. J. Response options and expectations of reward in decision-making: the differential roles of dorsal and rostral anterior cingulate cortex. Neuroimage 35, 979-988,

40 Kim, H. Y., Shin, Y. \& Han, S. The reconstruction of choice value in the brain: a look into the size of consideration sets and their affective consequences. J Cogn Neurosci $\mathbf{2 6}$, 810-824, doi:10.1162/jocn_a_00507 (2014).

41 Silvetti, M., Seurinck, R. \& Verguts, T. Value and prediction error estimation account for volatility effects in ACC: a model-based fMRI study. Cortex 49, 1627-1635, doi:10.1016/j.cortex.2012.05.008 (2013).

42 Hillman, K. L. \& Bikey, D. K. Neural encoding of competitive effort in the anterior cingulate cortex. Nature Neuroscience, 1290-1297 (2012).

43 Blanchard, T. C. \& Gershman, S. J. Pure correlates of exploration and exploitation in the human brain. Cogn Affect Behav Neurosci 18, 117-126, doi:10.3758/s13415-017-0556-2 (2018).

44 Sharot, T. \& Sunstein, C. R. How people decide what they want to know. Nat Hum Behav 4, 14-19, doi:10.1038/s41562-019-0793-1 (2020).

45 Kobayashi, K. \& Hsu, M. Common neural code for reward and information value. Proc Natl Acad Sci U S A 116, 13061-13066, doi:10.1073/pnas.1820145116 (2019).

46 Botvinick, M. M., Braver, T. S., Barch, D. M., Carter, C. S. \& Cohen, J. D. Conflict monitoring and cognitive control. Psychol Rev 108, 624-652 (2001).

47 Grinband, J. et al. The dorsal medial frontal cortex is sensitive to time on task, not response conflict or error likelihood. Neuroimage 57, 303-311,

doi:10.1016/j.neuroimage.2010.12.027 (2011).

48 Friston, K. A theory of cortical responses. Philos Trans R Soc Lond B Biol Sci 360, 815836, doi:10.1098/rstb.2005.1622 (2005).

49 Auer, P., Cesa-Bianchi, N. \& Fischer, P. Finite-time analysis of the multiarmed bandit problem. Machine Learning 47, 235-256 (2002).

50 Blanchard, T. C., Hayden, B. Y. \& Bromberg-Martin, E. S. Orbitofrontal cortex uses distinct codes for different choice attributes in decisions motivated by curiosity. Neuron 85, 602-614, doi:10.1016/j.neuron.2014.12.050 (2015).

51 White, J. K. et al. A neural network for information seeking. Nat Commun 10, 5168, doi:10.1038/s41467-019-13135-z (2019).

52 Alexander, W. H. \& Brown, J. W. Medial prefrontal cortex as an action-outcome predictor. Nat Neurosci 14, 1338-1344, doi:10.1038/nn.2921 (2011).

53 Alexander, W. H. \& Brown, J. W. Frontal cortex function as derived from hierarchical predictive coding. Sci Rep 8, 3843, doi:10.1038/s41598-018-21407-9 (2018). 
54 Vassena, E., Deraeve, J. \& Alexander, W. H. Surprise, value and control in anterior cingulate cortex during speeded decision-making. Nat Hum Behav, doi:10.1038/s41562019-0801-5 (2020).

55 Badre, D., Doll, B. B., Long, N. M. \& Frank, M. J. Rostrolateral prefrontal cortex and individual differences in uncertainty-driven exploration. Neuron 73, 595-607, doi:10.1016/j.neuron.2011.12.025 (2012).

56 Bromberg-Martin, E. S. \& Hikosaka, O. Midbrain dopamine neurons signal preference doi:10.1016/j.neuron.2009.06.009 (2009).

\section{(2011).}

58 Smith, D. V. \& Delgado, M. R. in Brain, Mapping: An Encyclopedic Reference Vol. 3 361-366 (Academic Press, 2015).

59 Chib, V. S., Rangel, A., Shimojo, S. \& O'Doherty, J. P. Evidence for a common representation of decision values for dissimilar goods in human ventromedial prefrontal cortex. J Neurosci 29, 12315-12320, doi:10.1523/JNEUROSCI.2575-09.2009 (2009).

60 Kim, H., Shimojo, S. \& O'Doherty, J. P. Overlapping responses for the expectation of juice and money rewards in human ventromedial prefrontal cortex. Cereb Cortex 21, 769776, doi:10.1093/cercor/bhq145 (2011).

61 Hampton, A. N., Bossaerts, P. \& O'Doherty, J. P. The role of the ventromedial prefrontal cortex in abstract state-based inference during decision making in humans. J Neurosci 26, 8360-8367, doi:10.1523/JNEUROSCI.1010-06.2006 (2006).

62 Boorman, E. D., Behrens, T. E., Woolrich, M. W. \& Rushworth, M. F. How green is the grass on the other side? Frontopolar cortex and the evidence in favor of alternative courses of action. Neuron 62, 733-743, doi:10.1016/j.neuron.2009.05.014 (2009).

63 McCoy, A. N., Crowley, J. C., Haghighian, G., Dean, H. L. \& Platt, M. L. Saccade reward signals in posterior cingulate cortex. Neuron 40, 1031-1040 (2003).

64 Pearson, J. M., Heilbronner, S. R., Barack, D. L., Hayden, B. Y. \& Platt, M. L. Posterior cingulate cortex: adapting behavior to a changing world. Trends Cogn Sci 15, 143-151, doi:10.1016/j.tics.2011.02.002 (2011).

65 Friston, K. et al. Active inference and epistemic value. Cogn Neurosci 6, 187-214, doi:10.1080/17588928.2015.1020053 (2015).

66 Samejima, K., Ueda, Y., Doya, K. \& Kimura, M. Representation of action-specific reward values in the striatum. Science 310, 1337-1340, doi:10.1126/science.1115270 (2005).

67 Smith, V. D., Rigney, A. E. \& Delgado, M. R. Distinct Reward Properties are Encoded via Corticostriatal Interactions. Scientific Reports, doi: DOI: 10.1038/srep20093 (2016).

68 Fiorillo, C. D., Tobler, P. N. \& Schultz, W. Discrete coding of reward probability and uncertainty by dopamine neurons. Science 299, 1898-1902, doi:10.1126/science. 1077349 (2003).

69 Cai, X., Kim, S. \& Lee, D. Heterogeneous coding of temporally discounted values in the dorsal and ventral striatum during intertemporal choice. Neuron 69, 170-182, doi:10.1016/j.neuron.2010.11.041 (2011). 
79870 Costa, V. D., Mitz, A. R. \& Averbeck, B. B. Subcortical Substrates of Explore-Exploit

799 Decisions in Primates. Neuron 103, 533-545 e535, doi:10.1016/j.neuron.2019.05.017

$800 \quad$ (2019).

$80171 \quad$ Humphries, M. D., Khamassi, M. \& Gurney, K. Dopaminergic Control of the

802 Exploration-Exploitation Trade-Off via the Basal Ganglia. Front Neurosci 6, 9,

803 doi:10.3389/fnins.2012.00009 (2012).

80472 O'Doherty, J. P. The problem with value. Neurosci Biobehav Rev 43, 259-268, doi:10.1016/j.neubiorev.2014.03.027 (2014).

73 Cogliati Dezza, I., Cleeremans, A. \& Alexander, W. Should we control? The interplay between cognitive control and information integration in the resolution of the exploration-exploitation dilemma. Journal of experimental psychology. General,

75 Daw, N. D. \& Doya, K. The computational neurobiology of learning and reward. Curr doi:10.1037/xge0000546 (2019). Opin Neurobiol 16, 199-204, doi:10.1016/j.conb.2006.03.006 (2006). Ross, S., Chaib-draa, B. \& Pineau, J. in Advances in Neural Information Processing Council.

831 Author Contribution: I.C.D. and W.A. designed and carried out the experiment and discussed the 832 computational modelling and fmri analysis. I.C.D. performed the fmri analysis and the model analysis.

833 I.C.D. and W.A. discussed and interpreted the data. I.C.D, A.C. and W.A. wrote the manuscript.

834 Competing Interests: The authors declare that they have no competing interests.

835 Supplementary Material: Supplementary text and Materials and Methods, Figures S1-3, Tables S1-S5, 836 References (1-6) accompanies this paper (bottom of this document). 


\title{
Supplementary Materials for
}

\author{
Distinct Value Systems for Reward and Information in Human Prefrontal Cortex \\ I. Cogliati Dezza, A. Cleeremens, W. Alexander
}

842

\section{This PDF file includes:}

849

850

Supplementary Text and Results

Figure S1 to S3

851 


\section{SUPPLEMENTARY TEXT AND RESULTS}

\section{Symmetrical activity in dual and single-value system}

855 Dual-value system simulations. To investigate whether correlations between information and reward are

856 expressed in the activity of a dual-value system, we ran 63 simulations of the gkRL model on our gambling-

857 task. The model parameters were selected in the range of those estimated in our sample. We computed

858 Information Gain $\left(-I_{t, j}(c)\right)$ and RelReward $\left(\mathrm{R} Q_{t+1, j}(c)\right)$ in order to simulate the activity associated with

859 the information system and the reward system. Next, we computed model activity in each contrast. To

860 simulate activity of the reward system in the reward contrast we ran a linear regression predicting

861 RelReward with RelReward as independent variable, while in the information contrast we ran a linear

862 regression predicting RelReward with Information Gain as independent variable. To simulate activity of

863 the information system in the reward contrast we ran a linear regression predicting Information Gain with

864 RelReward as independent variable, while in the information contrast we ran a linear regression predicting

865 Information Gain with Information Gain as independent variable. We averaged the beta coefficients across

866 simulations for each contrast and system and we ran a one-sample t test against zeros. As reported in Figure

867 1, averaged beta estimates in the reward and the information contrast were significantly different from zero

868 in both value systems. These results suggest that, even if reward and information are represented by distinct

869 and independent systems, reward and information signals influence activity in a opposite manner.

870 Single-value system simulations. In order to arrive at the overall value of an option, its subjective costs and

871 benefits are weighed against each other. It is generally assumed that the subjective value of an option is

872 modulated by factors that negatively impact the reward value, e.g., the level of effort required, decreases in

873 the probability of obtaining the reward, or longer delays before a reward is received. Similarly, subjective

874 costs may be modulated by the level of reward on offer: discounting rates for effortful, probabilistic or

875 delayed rewards are observed to vary as a function of reward level. In short, subjective costs and benefits

876 are not independent of one another.

877 We simulated a single-value RL model on our gambling task in which cost was modeled as the uncertainty

878 for selecting an option, consistent with uncertainty aversion observed in human decision-making, and 879 additionally modulated by the relative reward of an option :

$$
\operatorname{Cost}_{j}=I_{t, j}(c)-\mathrm{R} Q_{t+1, j}(c)(\mathrm{S} 1)
$$


Although uncertainty could be modeled in other ways (e.g., standard deviation of the outcome prediction for an option), for simplicity we adopt the same terms used in the gkrl model. For similar reasons, we model benefits as symmetrical to costs:

$$
\text { Value }_{j}=\mathrm{R} Q_{t+1, j}(c)-I_{t, j}(c)(\mathrm{S} 2)
$$

The assumption of a perfectly symmetrical value representation embodied in eq. S2 is likely incorrect -

886 decision-makers are frequently observed to overweight losses relative to gains, for example. Our rationale

887 for modeling a single-value system in this way is to demonstrate as directly as possible how such a system

888 responds to our analysis. Without accounting for the confound between reward and uncertainty/information,

889 the single-value system account makes the same predictions as the dual-system account.

\section{Accounting for information and reward confound}

891 To account for potential correlations between RelReward and Information Gain we ran the same set of 892 analyses explained above. However, we orthogonalized both RelReward and Information Gain using serial 893 orthogonalization ${ }^{1}$ to account for potential correlations between these two variables. Next, we simulate

894 activity for the reward system in the information contrast by entering the orthogonalized Information Gain

895 as independent $t$ variable, and to simulate activity for the information system in the reward contrast we 896 entered the orthogonalized RelReward. As shown in Figure 1, the symmetrical pattern of activity 897 disappeared.

898 The same procedure was conducted for the activity recorded from the single-value system model. In 899 contrast to the dual-value system simulations, serial orthogonalization of reward and 900 uncertainty/information does not eliminate effects for the alternate decision variable, i.e. we continued to 901 observe the symmetrical pattern of activity for the single-value RL model.

902 Information and reward confound in dual-value system generalizes to other decision-making 903 tasks

904 Here, we tested whether the symmetrical opposition in the dual-value system can be generalized to other 905 sequential decision-making tasks already published in the literature ${ }^{2,3}$. As in previous versions ${ }^{2,3}$, two cards 906 are displayed on each game and their reward magnitude is visible to the agent. The model has to decide 907 either to engage, which will lead to an economic decision between the two cards (engage cards), or to 
forage, which will lead to sample alternatives options from the back-up cards. The model has access to the reward magnitude of the back-up cards. Choosing to forage is associated with a cost (ranging between 0 and 3 points). We presented the agent with two conditions: High Information and Low Information. In High

911 Information, half of the back-up cards had lower values than those of the engaged options while the other

912 half had lower values. This condition has maximal uncertainty, i.e., the mean value of new cards obtained

913 through foraging was equally likely to be higher or lower than the mean value of the current cards.

914 Therefore, if the agent decides to forage it has no information on the actual value of the card that will be

915 selected. In the Low Information condition, all back-up cards could have higher or lower values with respect

916 to the engage options. This condition has minimum uncertainty, since the mean value of new cards was

917 guaranteed to be either higher or lower than the current mean card value. The task lasts 135 trials. On each

918 trial, the model computes the value of foraging (i.e., the mean reward of the back-up cards minus the cost

919 of foraging plus the uncertainty associated with the back-up cards: mean (Reward back-up cards) - cost +

920 sd (Reward back-up cards)) and the value of engaging (i.e., the mean reward of engage cards). The model

921 computes decision policies by entering both values into a softmax function. We classified model's choices

922 as HighReward (when choosing the option- forage or engage- associated with the highest mean reward),

923 LowReward (otherwise); HighInfoGain (when choosing forage in High Information condition) and

924 LowInfoGain (otherwise). Subsequently, we computed Information Gain as the value of foraging and

925 Reward as the value of engaging in order to simulate neural activity associated with the reward system and

926 information system. We then ran a first level analysis over the averaged value of engaging (value of

927 foraging) in the HighReward choices minus the average of reward values in the LowReward choices

928 (Reward Contrast); and the averaged value of engaging (value of foraging) in the HighInfoGain trials minus

929 the averaged value of engage (value of foraging) in LowInfoGain trials (Information Contrast). As already

930 shown with the simulation of our gambling task, activity associated with Reward and Information Contrast

931 are correlated in both value systems and both the reward dimension and information dimension are

932 represented in a symmetrically opposite manner within the two systems (Figure S1). These results

933 demonstrate that the confound in the representation of reward and information with value systems can be

934 generalized to other sequential decision-making tasks.

935 Information and reward system opposition in value-based choice in absence of symmetrical 936 opposition

937 Here, we show how functional opposition between reward and information systems in value-based choices

938 may be observed even in absence of clear symmetric opposition of activity, we simulated an effort-based

939 environment where rewards could be obtained only after exerting effort. In many effort-based paradigms ${ }^{4}$, 
940 subjects must choose between a small, default reward that requires little effort to obtain, or a larger reward

941 that requires greater effort, and consequently a chance of failing to perform the task adequately and not

942 receiving a reward. We adapted our RL model in order to simulate choices made by an agent performing

943 this task. In this implementation, the information value is equal to the entropy $\left(-p^{*} \log (p)\right.$; where $p$ is the

944 probability of successfully performing the task) resulting in the following value function:

$$
V_{t, j}(c)=Q_{t+1, j}(c) * p+p * \log (p(c)) * \omega
$$

946 We simulated the model across different ranges of effort and rewards. While the probability of the model

947 selecting the non-default option decreased with effort level (Figure S2A) and increased with relative reward

948 value (Figure S2B), consistent with research in this area, there was no correlation between the relative 949 reward value and effort level (Figure S2C). Finally, for the range of effort levels included in this simulation, 950 the level of effort correlated with the information value signal (Figure S2D). This result suggests that even 951 when activity indicating effort and relative reward can be dissociated in value-based decision-tasks, the 952 interpretation that the regions serve functionally opposed roles may be misguided.

\section{Logistic regression of subjects' behavior}

954 In order to investigate participants' behavior during the scanning session, we performed a logistic regression 955 for each participant over exploitative choices against the following normalized variables: highest 956 experienced reward (Highest Reward) and number of samples for the highest rewarded option ( $\mathrm{N}^{\mathrm{o}}$ samples).

957 The dependent variable had binary output \{exploitative choices $=1$; non-exploitative choices - or 958 exploration $=0$ otherwise $\}$. Exploitative choice trials were classified as those trials in which participants 959 chose the option in the first free-choice trial associated with the highest average of points collected during 960 the forced-choice task of the same game. Beta coefficients were collected for the entire group and a one 961 sample t-test was conducted as shown in the main text to test whether coefficients significantly differed 962 from 0 (Figure 2D).

\section{Parameter recovery, model comparison and model simulations}

964 We performed a parameter recovery analysis to estimate the degree of accuracy of the fitting procedure. To 965 do so, we simulated data from gkRL using the parameters obtained from the fitting procedure (true 966 parameters), and we fit the model to those simulated data to obtain the estimated parameters (fit 
parameters). We then ran a correlation for each pair of parameters ${ }^{5}$. This revealed high correlation coefficients for $\alpha\left(\mathrm{r}=0.8, p<10^{-3}\right), \beta\left(\mathrm{r}=0.8, p<10^{-3}\right), \omega(\mathrm{r}=0.6, p=0.006)$ and $\gamma(\mathrm{r}=0.6, p=0.002)$.

We additionally ran model comparisons to estimate the degree by which gkRL explained choice behavior

970 in our sample compared to a standard RL model- where only reward predictions influence choices. Negative

971 log likelihoods obtained during the fitting procedure were used to compute model evidence (the probability

972 of obtaining the observed data given a particular model). We adopted an approximation to the (log) model

973 evidence, namely the Bayesian Information Criterion (BIC) ${ }^{6}$ and we compared its estimate across different

974 models (fixed-effect comparison). Additionally, we used a random-effect procedure to perform Bayesian

975 model selection at group level ${ }^{7}$ where we computed an approximation of model evidence as -BIC/2. Model

976 comparison showed that the gkRL model was better able to explain participants' choice behavior compared

977 to a standard $\mathrm{RL}\left(\mathrm{xp}_{\mathrm{gkRL}}=1, \mathrm{BIC}_{\mathrm{gkRL}}=14354 ; \mathrm{xp}_{\text {standardRL }}=0, \mathrm{BIC}_{\text {standardRL }}=16990\right)$.

978 We simulated gkRL using the estimated free parameters. We then performed a logistic regression for each 979 simulation predicting gkRL choices (exploitative choices $=1$; non-exploitative choices - or exploration $=$ 980 0) with reward and number of samples as fixed effects. Exploitative choice trials were classified as those 981 trials in which the model chose the option in the first free-choice trial associated with the highest average 982 of points collected during the forced-choice task. Logistic regression was fitted for each individual 983 simulation and beta coefficients tested against zero. Reward and number of samples were significantly 984 impacting gkRL choices (both $\mathrm{p}<10^{-5}$ ) as observed in our human sample .

\section{Symmetrical opposition is also observed for expected reward values}

986 In GLM1, the reward regressor was defined as the relative reward value of the chosen option (RelReward).

987 We defined reward in this way based on previous research that showed vmPFC activity associated with the 988 relative reward value of the chosen option ${ }^{8}$. Here, we tested whether the symmetrical pattern was also 989 observed when computing reward as the expected value of the chosen option $\left(Q_{t+1, j}(c)\right.$; ExpReward). We 990 computed GLM1bis with ExpReward as a parametric modulator instead of the relative reward value. 991 ExpReward positively correlated with vmPFC (FWE $p<0.001$, voxel extent $=530$, peak voxel coordinates $992(-10,34,14), \mathrm{t}(19)=5.47)$ and negatively correlated with dACC $($ FWE $p=0.001$, voxel extent $=205$, 993 peak voxel coordinates $(8,22,40), \mathrm{t}(19)=5.47)$ suggesting that the symmetrical pattern was also observed 994 when considering expected rewards and not just their relative estimates. 

reward value

997 Here, we tested whether the reward signal computed in vmPFC was best described by the relative reward

998 value of the chosen option (our RelReward regressor) or by the expected reward value of the chosen option

999 (ExpReward). We computed GLM9 with ExpReward (controlling for RelReward) and GLM10 with

1000 RelReward (controlling for ExpReward) as parametric modulators. Activity in vmPFC positively

1001 correlated with relative reward after controlling for ExpReward (FWE $p<0.001$, voxel extent $=1829$, peak

1002 voxel coordinates $(-6,52,14), \mathrm{t}(19)=7.21)$. However, vmPFC activity associated with ExpReward

1003 disappeared after controlling for RelReward suggesting that vmPFC encodes the relative reward value of

1004 the chosen option as previously suggested ${ }^{8}$.

\section{Additional reward covariates}

1006 Here, we investigated the role that additional reward covariates can play in modulating vmPFC activity. We

1007 individuated 5 additional covariates: the maximum value of 3 decks (Max Value), the minimum value of 1008 the 3 decks (Min Value), the reward value variation for the chosen option (Standard Deviation), the averaged 1009 value of the 3 decks (Averaged Value), the value of the chosen option minus the value of the best second 1010 option (Chosen-Second). We first computed the correlations with Reward (Table S5). Standard Deviation 1011 and Chosen-Second showed really high correlation with RelReward therefore were not considered any 1012 further. For the remaining variables, we entered them as parametric modulators in a GLM11 together with 1013 Reward allowing to compete for variance. We then extracted vmPFC ROI from GLM2 (to avoid that reward 1014 activity might interfere with ROI identification) and we computed the averaged beta estimates for each 1015 covariate. Next, we compared these betas to the betas estimated in vmPFC ROI for RelReward. Results 1016 showed that the betas for RelReward were significantly higher than the betas for Max Value $(\mathrm{p}<0.05)$, 1017 Min Value $(\mathrm{p}<0.05)$, Averaged Value $\left(\mathrm{p}<10^{-3}\right)$ suggesting that the majority of the variance in vmPFC was 1018 accounted by our RelReward.

1019 dACC encodes the value of information after controlling for the expected reward value

1020 The analyses reported in the main text clearly suggest that dACC encodes the value of information. 1021 However, in GLM4 the activity associated with the value of information was controlled by the variance 1022 explained by the relative reward value. It is possible that the variance could only account for the 1023 "relativeness" instead of the reward itself. Therefore, we ran an additional analysis to investigate activity 1024 associated with Information Gain after controlling for ExpReward (GLM4bis). Results showed that 
1025 Information Gain was positively correlated with dACC after controlling for ExpReward (FWE $p<0.001$, 1026 voxel extent $=640$, peak voxel coordinates $(10,30,58), \mathrm{t}(19)=6.87)$ suggesting that whether we control

1027 for expected reward or their relative estimates activity associated with Information Gain was still expressed 1028 in dACC.

\section{Dissociable regions for Reward and Information}

1030 While our results from GLMs $3 \& 4$ demonstrate that activity in vmPFC \& posterior cingulate is explained 1031 by RelReward after controlling for Information Gain, and activity in AACC \& anterior insula is explained 1032 by Information Gain after controlling for RelReward, these analyses do not allow us to conclude that one 1033 set of regions is specific to reward while the other is specific to information (i.e., while we can say the, for 1034 example, RelReward is different than 0, and Information Gain is not different than 0, we cannot say 1035 RelReward is different than Information Gain). In order to do so, we directly compare the beta weights 1036 estimated for RelReward (after orthogonalizing with respect to Information Gain) from GLM3 and the beta 1037 weights estimated for Information Gain (orthogonalized with respect to Reward) from GLM4 using a 1038 paired-sample t-test. We find clusters of activity in $\operatorname{vmPFC~(as~reported~in~the~main~text),~posterior~cingulate~}$ 1039 (FWE $p<0.001$, voxel extent $=1493$, peak voxel coordinates $(-14,-48,36), \mathrm{t}(19)=6.02)$ and putamen $1040 \quad($ FWE $p<0.001$, voxel extent $=920$, peak voxel coordinates $(24,10,-8), t(19)=6.13)$ in which Reward $>$ 1041 Information Gain (Figure S3A), indicating that these regions are specifically involved in reward processing, 1042 while a significant cluster is observed in AACC (as reported in the main text), right insula (FWE $p<0.05$, 1043 voxel extent $=157$, peak voxel coordinates $(34,24,-6), \mathrm{t}(19)=4.89))$ and dlPFC $(\mathrm{FWE} p<0.05$, voxel 1044 extent $=158$, peak voxel coordinates $(48,32,32), \mathrm{t}(19)=4.89)$ for (negative) Information Gain $>$ 1045 RelReward (Figure S3B), indicating that dACC is specifically involved in representing uncertainty. 


\section{SUPPLMENTARY REFERENCES}

10481 Mumford, J. A., Poline, J. B. \& Poldrack, R. A. Orthogonalization of regressors in FMRI models. PLoS One 10, e0126255, doi:10.1371/journal.pone.0126255 (2015).

1051

1052

1053

1054

1055

1056

1057

1058

1059

2 Shenhav, A., Straccia, M. A., Botvinick, M. M. \& Cohen, J. D. Dorsal anterior cingulate

1060

1061

1062

1063

1064 and ventromedial prefrontal cortex have inverse roles in both foraging and economic choice. Cogn Affect Behav Neurosci 16, 1127-1139, doi:10.3758/s13415-016-0458-8 (2016).

3 Kolling, N., Behrens, T. E., Mars, R. B. \& Rushworth, M. F. Neural mechanisms of foraging. Science 336, 95-98, doi:10.1126/science.1216930 (2012).

4 Hogan, P. S., Galaro, J. K. \& Chib, V. S. Roles of Ventromedial Prefrontal Cortex and

1065 Anterior Cingulate in Subjective Valuation of Prospective Effort. Cereb Cortex 29, 4277 4290, doi:10.1093/cercor/bhy310 (2019).

5 Wilson, R. C. \& Collins, A. G. Ten simple rules for the computational modeling of behavioral data. Elife 8, doi:10.7554/eLife.49547 (2019).

6 Schwarz, G. Estimating the dimension of a model. Ann. Stat 6, 461-464 (1978).

7 Stephan, K. E., Penny, W. D., Daunizeau, J., Moran, R. J. \& Friston, K. J. Bayesian model selection for group studies. Neuroimage 46, 1004-1017, doi:10.1016/j.neuroimage.2009.03.025 (2009).

1066

1067

1068

1069 
Figure S1. Correlated activity in foraging task.

1071

A

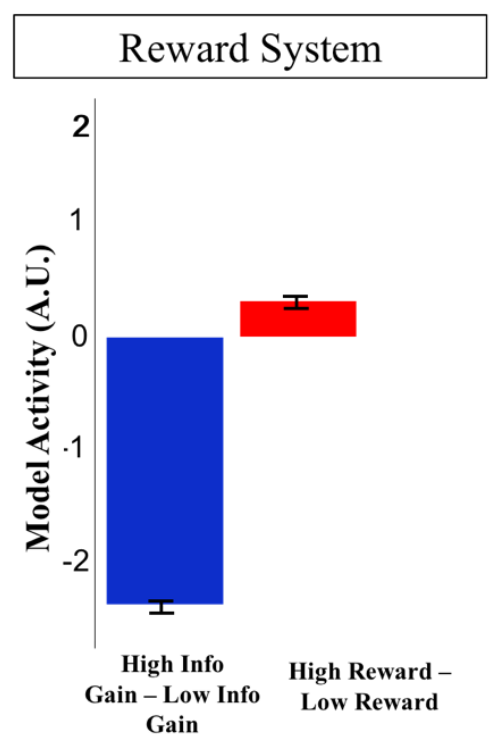

B

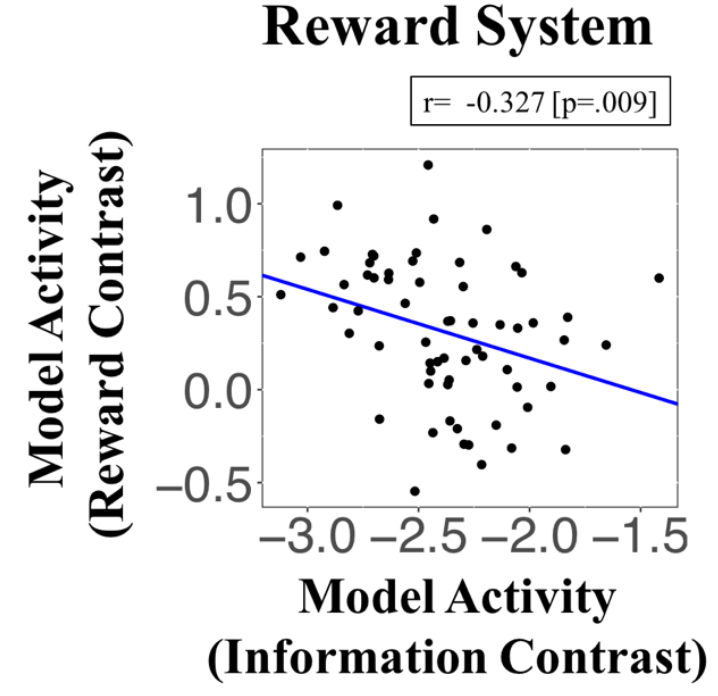

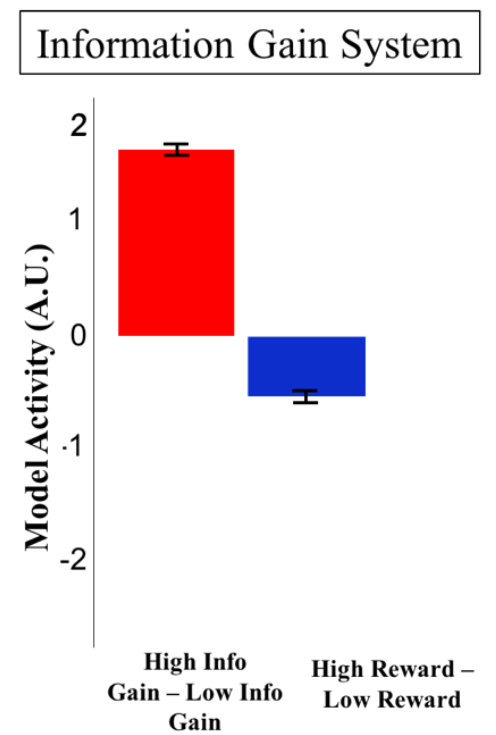

C

Information Gain System

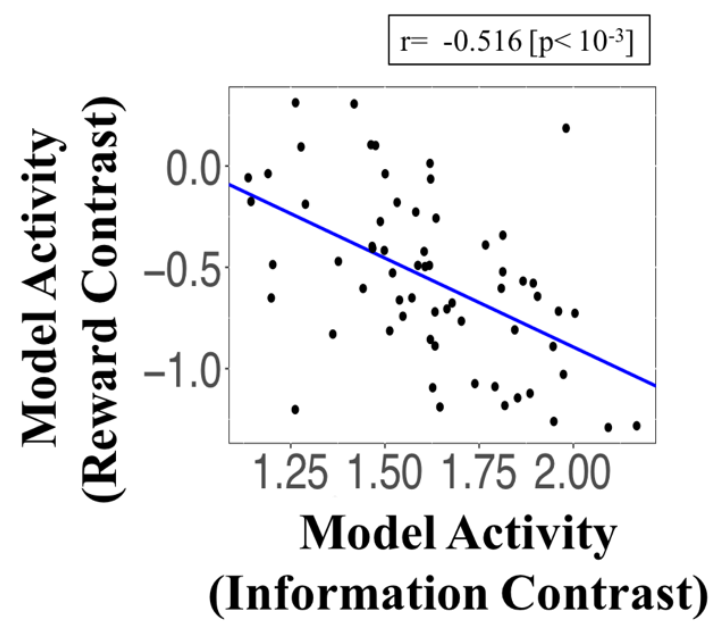

1072

1073

1074

1075

1076

1077

1078
A) Simulating a dual value system on the sequential decision-making task adopted by ${ }^{3}{ }^{2}$. Despite the independence of information and reward systems, the systems' activity are correlated: optimizing information is associated with decreased activity in the reward value system, and optimizing reward is associated with decreased activity in the information value system. Activity within the (B) reward system and $(\mathbf{C})$ the information system is negatively correlated across independent model simulations. 
1079 Figure S2. Functional opposition between $v m P F C$ and $d A C C$ in absence of symmetrical 1080 opposition.
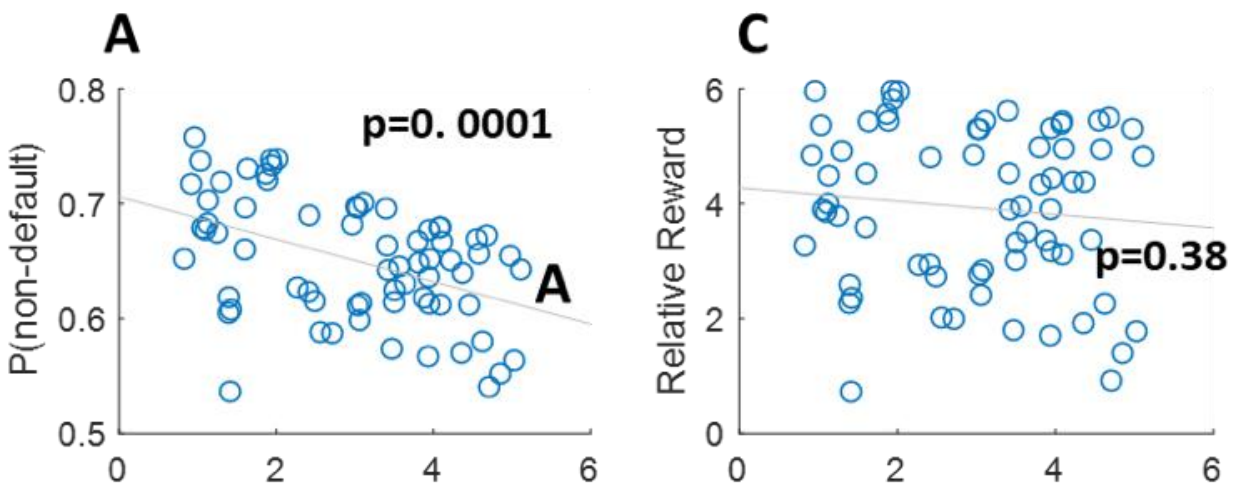

1081
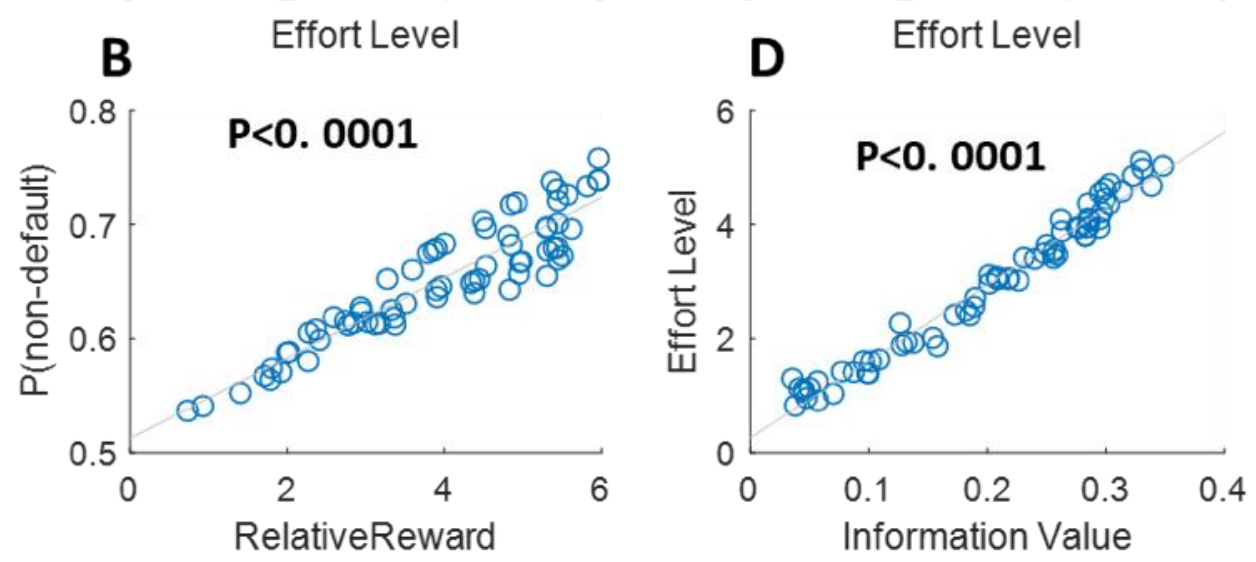

1082

1083

Probability of the model selecting the non-default option across effort levels (A) and its relative reward values $(\mathbf{B})$. Correlation between relative reward value and effort levels $(\mathbf{C})$. Correlation between

1084 information value and effort levels $(\mathbf{D})$.

1085

1086 
1087 Figure S3. Domain specificity in vmPFC and $d A C C$.

A

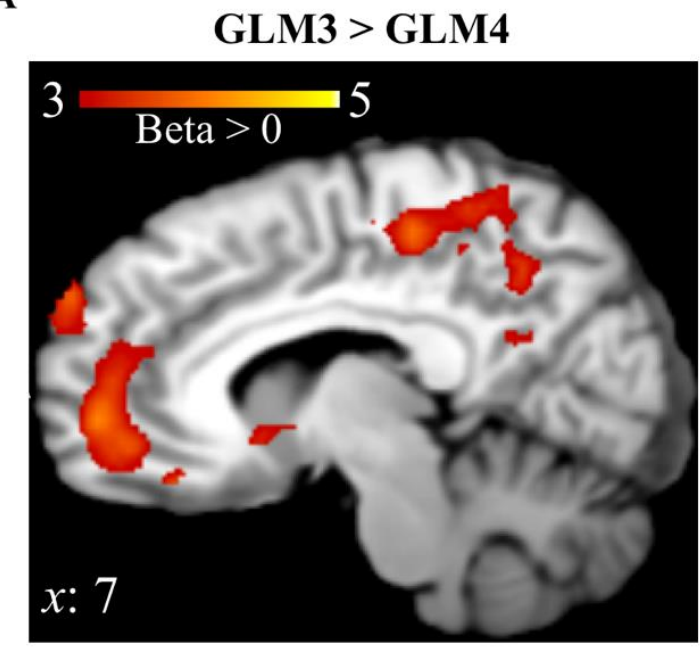

B

\section{GLM4 > GLM3}

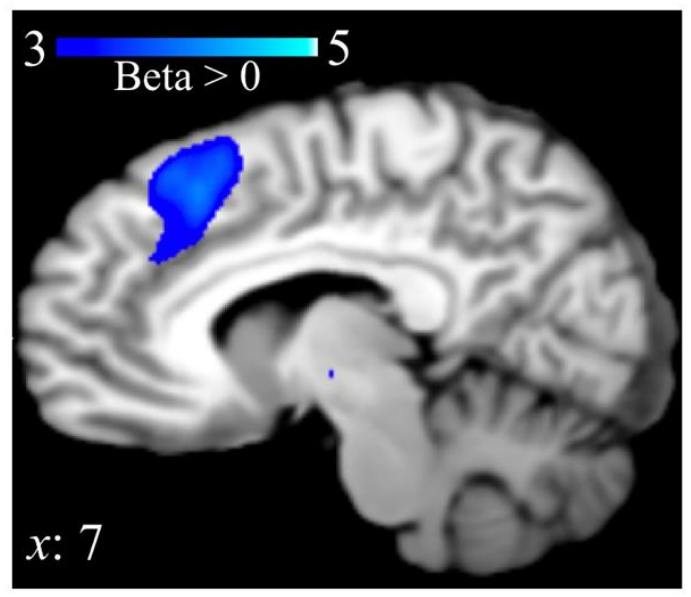

A paired t-test between GLM3 and GLM4 shows A) specificity for reward (and not for information) in

1090 vmPFC, and $\mathbf{B}$ ) for information (and not for reward in dACC). 
1092 Tables:

1093 Table S1. vmPFC and dACC opposition across different decision-making contexts.

\begin{tabular}{|c|c|c|}
\hline STUDIES & VMPFC & dACC \\
\hline Blair et al. 2006 & Greater reward & Lesser punishment \\
\hline Kolling et al. 2012 & Value of engaging & Value of foraging \\
\hline Boorman et al. 2013 & Immediate reward & Long-term reward \\
\hline Chen et al. 2014 & $\begin{array}{c}\text { Positive correlation with creativity } \\
\text { questionnaire }\end{array}$ & $\begin{array}{l}\text { Negative correlation with } \\
\text { creativity questionnaire }\end{array}$ \\
\hline Kolling et al. 2014 & Reduced risk & Increased risk \\
\hline Skvortsova et al. 2014 & Expected reward & Negative Expected reward \\
\hline Becker et. al. 2016 & Exploit reward & Switch to alternatives \\
\hline Sinha et al. 2016 & $\begin{array}{l}\text { Early decrease, later increase } \\
\text { during stress manipulation }\end{array}$ & $\begin{array}{l}\text { Early increase, later decrease } \\
\text { during stress manipulation }\end{array}$ \\
\hline Shenhav et al. 2016 & Choice ease & Choice difficulty \\
\hline Delaveau et al. 2017 & Default Mode Network & Task Positive Network \\
\hline Arulpragasam et al. 2018 & Subjective value & Negative Subjective value \\
\hline Suarez-Jimenez et al. 2018 & Learning danger & Approaching danger \\
\hline
\end{tabular}

1094

1095 The table shows a selection of studies that report the symmetric opposition between vmPFC and dACC in 1096 value-based decision-making. 
Table S2. Model estimated parameters from participants' behavior.

\begin{tabular}{|c|c|c|c|c|}
\hline & \multicolumn{4}{|c|}{ gkRL } \\
\hline Participants & $\alpha$ & $\beta$ & $\omega$ & $\log (\gamma)$ \\
\hline Number1 & 0.004 & 7.916 & 0.457 & -1.895 \\
\hline Number2 & 0.151 & 0.443 & 2.723 & 0.098 \\
\hline Number3 & 0.684 & 0.189 & 17.52 & -13.81 \\
\hline Number4 & 0.495 & 0.358 & 33.333 & -1.61 \\
\hline Number5 & 0.518 & 0.201 & 12.980 & -1.46 \\
\hline Number6 & 0.459 & 0.168 & 48.663 & -13.81 \\
\hline Number7 & 0.528 & 0.147 & 9.181 & -0.748 \\
\hline Number8 & 0.593 & 0.158 & 24.743 & -1.84 \\
\hline Number9 & 0.658 & 0.214 & 33.661 & -1.84 \\
\hline Number10 & 0.054 & 1.819 & 1.714 & 0.138 \\
\hline Number11 & 0.761 & 0.155 & 31.631 & -18.07 \\
\hline Number12 & 0.687 & 0.142 & 1.114 & -0.003 \\
\hline Number13 & 0.660 & 0.125 & 29.913 & -2.64 \\
\hline Number14 & 0.393 & 0.321 & 17.714 & -19.94 \\
\hline Number15 & 0.23 & 0.338 & 4.808 & -0.134 \\
\hline Number16 & 0.269 & 0.791 & 7.528 & -0.425 \\
\hline Number17 & 0.046 & 0.244 & 3.032 & -2.43 \\
\hline Number18 & 0.269 & 0.208 & 9.174 & -0.178 \\
\hline Number19 & 0.011 & 1.562 & 0.508 & -0.642 \\
\hline Number20 & 0.012 & 2.084 & 0.26 & -0.080 \\
\hline Total & 0.374 & 0.879 & 14.53 & 4.07 \\
\hline & $0.264)$ & $0.176)$ & $(1.76)$ & $(6.51)$ \\
\hline
\end{tabular}


Table S3. GLMs for fMRI data.

NAME REGRESSORS

\begin{tabular}{|c|c|}
\hline GLM0 & [Highest Reward choice; Lower Reward choice] \\
\hline GLM1 & [First free choice; $R Q_{t+1, j}(c) ; 24$ motion regressors] \\
\hline GLM1bis & [First free choice; $Q_{t+1, j}(c) ; 24$ motion regressors] \\
\hline GLM2 & [First free choice; $-I_{t, j}(c) ; 24$ motion regressors] \\
\hline GLM3 & [First free choice; $-I_{t, j}(c) ; R Q_{t+1, j}(c) ; 24$ motion regressors] \\
\hline GLM4 & [ First free choice; $R Q_{t+1, j}(c) ; I_{t, j}(c) ; 24$ motion regressors] \\
\hline GLM4bis & [ First free choice; $Q_{t+1, j}(c) ;-I_{t, j}(c) ; 24$ motion regressors] \\
\hline GLM5 & [ First free choice; Instrumental Info $(c) ;-I_{t, j}(c) ; 24$ motion regressors] \\
\hline GLM6 & [ First free choice; $-I_{t, j}(c) ;$ Instrumental Info $(c) ; 24$ motion regressors] \\
\hline GLM7 & [Defulat Option; Switch Option] \\
\hline GLM8 & [ First free choice; $P\left(c / V_{t, j}\left(c_{i}\right)\right) ; 24$ motion regressors] \\
\hline GLM9 & [ First free choice; $R Q_{t+1, j}(c) ; Q_{t+1, j}(c) ; 24$ motion regressors] \\
\hline GLM10 & [ First free choice; $Q_{t+1, j}(c) ; R Q_{t+1, j}(c) ; 24$ motion regressors] \\
\hline GLM11 & [ First free choice; $R Q_{t+1, j}(c) ; \operatorname{Max} Q_{t+1, j} ; \operatorname{Min} Q_{t+1, j} ;$ mean $Q_{t+1, j} ; 24$ motion regressors] \\
\hline
\end{tabular}

1105 The table shows the 13 GLMs adopted in the fMRI data analysis all referring to activity associated with the 1106 onset of the first-free choice trial. GLM0 and 7 are the univariate analysis, whereas the other GLMs relate 1107 with the model-based analysis. 
1109 Table S4. Brain activity no reported in the text.

\begin{tabular}{|c|l|l|}
\hline GLM & \multicolumn{1}{|c|}{ ROI } & $\begin{array}{l}\text { Statistics } \\
\text { (cluster level } \\
\text { correction) }\end{array}$ \\
\hline GLM0 & PCC (8, -44, 28) & $\begin{array}{l}p<0.001 \\
p<0.001 \\
p=0.037\end{array}$ \\
\hline Highest reward - Lower Reward & mCC (-6, -22, 42) & $p<0.001$ \\
\hline GLM1 (Beta $>0)$ & PCC (-8, -50, 28) & $p<0.01$ \\
\hline & right aInsula (34, 22, -8) & $p<0.05$ \\
GLM2 (Beta $>0)$ & left aInsula (-34, 20, 6) & $p=0.001$ \\
\hline GLM2 (Beta $<0)$ & right dLPFC (42, 16, 40) & $p<0.001$ \\
\hline GLM3 (Beta $>0)$ & PCC (-10, -34, 46) & $p<0.001$ \\
\hline GLM4 (Beta $>0)$ & PCC (-2, -50, 26) & $p<0.05$ \\
& right aInsula (34, 22, -8 ) & $p<0.05$ \\
\hline GLM8 $($ Beta $>0)$ & left aInsula (-38, 18, -10) & $p<0.002$ \\
\hline
\end{tabular}

The table shows brain activity not reported in the main text. PCC: Posterior Cingulate Cortex; mOFC: 
1115 Table S5. Correlation of covariates with relative reward value.

1116

\begin{tabular}{|c|c|c|c|c|c|}
\hline Subject & Max Value & Min Value & $\begin{array}{l}\text { Standard } \\
\text { Deviation }\end{array}$ & $\begin{array}{l}\text { Averaged } \\
\text { Value }\end{array}$ & Chosen-Second \\
\hline 1 & 0.135 & 0.135 & 0.718 & -0.123 & 0.938 \\
\hline 2 & 0.333 & 0.333 & 0.657 & -0.013 & 0.889 \\
\hline 3 & 0.115 & 0.115 & 0.672 & -0.116 & 0.917 \\
\hline 4 & 0.504 & 0.504 & 0.574 & -0.061 & 0.884 \\
\hline 5 & 0.162 & 0.162 & 0.649 & -0.282 & 0.924 \\
\hline 6 & 0.441 & 0.441 & 0.586 & -0.048 & 0.892 \\
\hline 7 & -0.082 & -0.082 & 0.65 & -0.461 & 0.938 \\
\hline 8 & 0.539 & 0.539 & 0.54 & 0.054 & 0.881 \\
\hline 9 & 0.355 & 0.355 & 0.488 & -0.049 & 0.882 \\
\hline 10 & 0.34 & 0.34 & 0.649 & -0.163 & 0.927 \\
\hline 11 & -0.025 & -0.025 & 0.603 & -0.319 & 0.920 \\
\hline 12 & 0.386 & 0.386 & 0.551 & -0.105 & 0.920 \\
\hline 13 & 0.646 & 0.646 & 0.547 & 0.074 & 0.881 \\
\hline 14 & 0.327 & 0.327 & 0.584 & -0.13 & 0.914 \\
\hline 15 & 0.511 & 0.511 & 0.646 & 0.03 & 0.890 \\
\hline 16 & 0.396 & 0.396 & 0.638 & -0.016 & 0.896 \\
\hline 17 & 0.474 & 0.474 & 0.709 & 0.018 & 0.897 \\
\hline 18 & 0.327 & 0.327 & 0.714 & 0.101 & 0.918 \\
\hline 19 & 0.101 & 0.101 & 0.681 & -0.247 & 0.923 \\
\hline 20 & 0.117 & 0.117 & 0.68 & -0.14 & 0.941 \\
\hline 21 & 0.143 & 0.143 & 0.595 & -0.062 & 0.900 \\
\hline
\end{tabular}

